\title{
Image Texture and Fourier Analysis in a Polar Spatial Frequency System: methods illustration
}

\author{
Erin Fowler ${ }^{1}$, Steven Eschrich ${ }^{2}$, and John Heine ${ }^{1, *}$
}

${ }^{1}$ Department of Cancer Epidemiology, H. Lee Moffitt Cancer Center \& Research Institute, 12901 Bruce B Downs Blvd., Tampa FL, 33612 (MCC)

${ }^{2}$ Department of Biostatistics and Bioinformatics, MCC

*Corresponding Author: john.heine@moffitt.org

\begin{abstract}
We illustrate the connection between image-texture and defined regions in a polar spatial frequency coordinate system. This is provided within the context of describing a non-parametric method for characterizing twodimensional spectra of gray scaled images, described in part previously. The spectrum is divided into concentric radial bands of fixed bandwidth. Each band is summarized, producing a set of measures characterizing the image as a function of radial spatial frequency. This set of measures has a parallel multiscale description as texture in the image domain. This approach was modified to capture texture with directional attributes. This is a data reduction technique that gives a full accounting of the image variance as function of image texture and direction indexed to regions in the polar frequency plane. Application examples include analyzing the summarized spectrum graphically, making inter/intra image spectral comparisons, and evaluating spectral models. Simulated noise images, an image of peppers, and a gray scale image with well-defined linear structure were used to illustrate the principles.
\end{abstract}

Keywords: Image Texture, Multiscale Filtering, Fourier Analysis, Polar Coordinates, Data Reduction, Instructional 


\section{Introduction}

Image analysis techniques are used in numerous settings. Examples include biomedical research [1-3], remote sensing [4, 5], process quality control [6], and facial recognition [7]. In biomedical research, classification is a recurrent methodology, where image measurements (features) can be used to separate individuals (or images) into two [8] or more classes [9]. Similarly, risk prediction is an epidemiologic variant of classification that can include image measurements [10]. Examples of other image processing applications include remote sensing for monitoring spatial-temporal changes in wetlands [4] and mapping natural disasters [5], surface defect detection $[11,12]$, evaluating concrete structural condition [13], identifying individuals with automated facial recognition techniques [7], and modeling to understand the spectral characteristics of natural images [14-16].

Developing features from digital images can take the form of texture analysis, as illustrated in mammography [10, 17], lung imaging [18], neurologic magnetic resonance [19], and surface defect analysis [11]. Texture features can be investigated by applying filters to the image with a convolution operation or equivalently with a multiplication operation in the Fourier domain (FD) followed by Fourier inversion (see Appendix for the relevant Fourier relationships provided in Eqs. [A1-A8]). Although there are other methods for evaluating texture [17], this report is restricted to texture derived from filtering. Measurements can be made globally, such as the variance calculated over the entire field of view (FOV). Alternatively, measurements can be made locally; local measures can be summarized over the FOV. Methods in this report fall within the global measurement approach (i.e. capturing field effects) because they are derived in the FD. The equivalent FD interpretation of texture is often not addressed in detail in contrast with the spatial domain description.

There is a common property between visually disparate images, relevant to the methods presented in this report. The spectra of mammograms [20-22], natural scenes [14-16], and the earth's topography [23] are well approximated with an inverse power law (i.e. exhibiting fractal characteristics or statistical self-similarity). The spectra of visual art also follows this form [24]. Under this form, the power spectrum understudy is a radially symmetric function of distance measured from the origin in the spatial FD. An image with this characteristic would not exhibit texture with directional preference such as striations restricted to specific orientations. Details describing the nature of such images and how they can be formed are discussed by Schroeder [25]. In contrast, it has been observed that the spectra for natural scenes may not be isotopically distributed when considering spectra as a function of orientation [14]. Similarly, we have shown that the spectra of mammograms exhibit asymmetries when analyzing region sizes much smaller than the image dimension [26]. Whether the spectra of such disparate images are radially symmetric is not the focus of this report. Nevertheless, the methods discussed in this report can be used to evaluate spectral power law assumptions, as illustrated. 
The purpose of this report is to illustrate the connection between image texture and regions in the FD. This is provided within the context of describing a non-parametric method for characterizing the spectra of twodimensional images. This approach divides the power spectrum of a given image into concentric bands of constant radial bandwidth, neglecting angular dependencies, described previously [17, 22, 27]. Summation over a given band produces a measure indexed to its specific location in the radial frequency plane and bandwidth. The set of bands can be considered as radial filter bank when viewed in the image domain. In this report, the radial characterization was modified to capture texture with directional attributes and incorporated it into the indexing scheme. The approach is presented as a FD data reduction technique that gives a full accounting of the power spectrum, or equivalently the image variance, with a radial spatial frequency indexing scheme related to texture or spatial scale and direction. Although the approach is non-parametric, it can be converted to a parametric spectral modeling technique under certain assumptions. We also show how the technique can be improved to give better spatial characteristics. In describing this technique, we rely heavily on radial spatial frequencies. The main concepts are presented by filtering noise images, an image of peppers, and another gray scaled image with pronounced linear structure in well-defined directions.

\section{Methods}

\subsection{Overview}

Due to the objectives and the illustrative nature of this report, the presentation format does not follow that of a standard research article with an experimental methods and results sections. For continuity, we first outline the format, objectives, methods used to illustrate the central concepts, and describe the images analyzed in this report. To make the work self-contained, a brief overview of the Fourier transform (FT) is provided in the Appendix for those not familiar with this type of analysis (see Eq. [A1] - Eq. [A8]). Specific Fourier relationships used in this report are also described in this section. Illustrations are presented in a hierarchical order leading to the twodimensional data reduction technique indexed to spatial scale and direction. This is followed by demonstrating how the approach can be used for parametric spectral modeling and providing methods for improving the spatial characteristics of the various filters.

More specifically, the results (Section 3) is comprised of subsections that sequentially mirror the respective illustrations. In Section 3.1, the connection between texture and well-defined regions in the Fourier domain (FD) is illustrated with disk filters (i.e. pillbox type FD filters with unit height) applied to simulated twodimensional random noise images. These are lowpass filters with exact upper radially symmetric cutoff frequencies. By successively reducing the cutoff frequency by $1 / 2$ (i.e. reducing the pillbox spatial frequency radius), applying the respective filter to the FT of noise followed by Fourier inversion, different textures were 
generated. In Section 3.2, the corresponding spatial domain filter kernels for the disk filters were derived numerically with the inverse FT and compared with the theoretical kernels; these expressions were used to derive the radial band filter kernels. In Section 3.3, the central analysis of dividing the Fourier power spectrum into concentric bands is described. These examples show the connection between specific coordinates in the spatial frequency plane and the corresponding two-dimensional radial sinewaves in the image domain. In Section 3.4, texture captured by a given radial band is described by considering the alternative filtering (convolution) operation in the spatial domain applied to noise and gray scaled images; the corresponding image domain bandpass filter kernels were derived in closed form. These theoretical expressions were compared with the corresponding numerically generated kernels. In Section 3.5, the radial band analysis was modified to capture texture with directional attributes by restricting radial bands to specific arcs. As a more detailed analysis to connect the Fourier indexing to scale and direction in the image domain, a gray scaled image with strong directional characteristics was analyzed in detail; this illustration also demonstrates how the approach can be used to evaluate radial spectral symmetry. In Section 3.6, the band analysis was used to evaluate spectral modeling through integration. A specific model that can approximate an inverse power law was used to analyze a gray scaled image of peppers. In Section 3.7, improvements to the technique were developed. Because the approach uses exact and relatively narrow frequency specification, spatial localization suffers. We show how to modify the band analysis to improve spatial localization.

\subsection{Fourier Relationships and Coordinate Systems}

Although the approach sums power (Eq. [A8]) in the FD, there is a corresponding spatial measure for each band that can be derived by convolving the image (see Eq. [A2] - Eq. [5]) with the respective filter kernel and then calculating the variance. The inverse FT of the filter, $H\left(f_{x}, f_{y}\right)$, (see Eq. [A9]) gives the filter kernel referenced as $h(x, y)$, where $\left(f_{x}, f_{y}\right)$ and $(x, y)$ are spatial frequency and spatial Cartesian coordinates, respectively. The spatial extent of $\mathrm{h}(\mathrm{x}, \mathrm{y})$ depends on its localization in the FD. The Fourier scaling property indicates a contraction in one domain corresponds with a broadening in the other domain (i.e. $\mathrm{h}(\mathrm{x}, \mathrm{y})$ spreads out). When taking the global measurement approach in the spatial domain, it is our premise that this tradeoff is acceptable. It follows, that we can study exact frequency specification when the image view is not the focus. Techniques described in this report may be used for analyzing phenomena better characterized as field (global) effects rather than localized anomalies.

Digital images are represented by samples, referred to as pixels. The discrete FT gives sample points in frequency domain. In theory, the center to center pixel spatial distance is related to the highest resolvable spatial 
frequency. Starting with a one-dimensional discrete signal, we let d equal the distance between signal samples measured in $\mathrm{mm}$ for this report (this is equivalent to the center to center pixel separation). The spatial sampling

frequency is given by $\mathrm{s}=\mathrm{d}^{-1}$ per mm. To avoid aliasing, the sampling frequency (Nyquist Criterion) is defined as, $s=2 \times f_{c}$, where $f_{c}$ is the highest resolvable spatial frequency in the signal [28]. Combining these expressions gives $f_{c}=1 / 2 \times d^{-1}$. This principle can be extended to two-dimensions [29]; with isotropic spatial sampling in a Cartesian system, the spectral content of given image is defined within these bounds: $\left(\left|f_{c}\right|,\left|f_{c}\right|\right)$. We note in theory, a signal (or image) cannot be limited in extent in both domains simultaneously, although in applied work signals are well approximated with this characteristic [30].

Both Cartesian and polar coordinates were used for the spatial frequency plane. To aid the radial frequency description, $\left(f_{x}, f_{y}\right)$ were expressed in polar coordinates, $(f, \phi)$, with the standard coordinate system change given by

$$
\mathrm{f}=\sqrt{\mathrm{f}_{\mathrm{x}}^{2}+\mathrm{f}_{\mathrm{y}}^{2}} \text {, with } \mathrm{f}_{\mathrm{x}}=\mathrm{f} \times \cos (\phi) \text { and } \mathrm{f}_{\mathrm{y}}=\mathrm{f} \times \sin (\phi) \text { for } 0 \leq \phi \leq 2 \pi \text {, }
$$

where $\mathrm{f}$ is a radial spatial frequency variable.

\subsection{Study Images and Filters}

The work analyzed two-dimensional random noise images, an image of peppers, and a gray-scaled image titled Crowd Walking in Alley [31], referenced as CWA in this report (appears to be a World Trade Center station). Zero mean random noise (white) images were generated with normally distributed random variables with these dimensions: $1001 \times 1001$ pixels (double precision). These were processed with filters constructed in the FD that were also $1001 \times 1001$ pixels (double precision). In the numerical manipulations, where units aided the illustrations, we used $\mathrm{d}=70 \mu \mathrm{m}$ ( or $0.07 \mathrm{~mm}$ ) giving $\mathrm{f}_{\mathrm{c}} \approx 7.14$ cycles $/ \mathrm{mm}$, following from our work in mammography [27] unless otherwise noted. The peppers image is in 24-bit red-green-blue (RGB) format with $512 \times 512$ pixels. We used the $\mathrm{G}$ (green) component, resulting in an 8-bit gray scaled image. We also trimmed one pixel from half of its perimeter (i.e. bottom and one side), reducing it to $511 \times 511$ pixels, and mean centered the resultant image. The CWA dimensions are $5468 \times 3645$ pixels with three channels, where each channel has the same 8-bit image. We used one $(\mathrm{G})$ channel, trimmed one pixel in the x-direction (i.e. $5467 \times 3645$ pixels), and mean centered the resulting image. We used CWA because it has pronounced linear structure in specific directions. These gray scaled images were processed with FD filters (double precision) constructed with the same respective dimensions. Although not a requirement, odd sized images permits centering the FT symmetrically in the FD. 


\section{Results}

\subsection{Image Texture and the Fourier Domain}

The connection between image texture and regions in the FD was developed with disk (pillbox) filters applied to noise, illustrated in Figure 1. White noise has a uniform power spectrum, and therefore lacks spatial correlation (no structure). When applying a given filter (bandpass or lowpass in our applications) to an arbitrary image, certain frequency components are modified. In our application, components are attenuated (blocked) making other components more pronounced in the output image, if present initially. Filtering noise induces spatial correlation in the output image, observed as spatially uniform image texture, dependent upon the filter profile. In the following illustrations, texture was generated by filtering white noise. In general, overlapping textures can exist in non-simulated images that may not be spatially stationary, very different from filtered white noise. Nevertheless, these stationary idealizations are useful for illustration purposes.

Disk filters were applied to the FT of two-dimensional noise images followed by Fourier inversion. The top row of Figure 2 shows three filters in the FD with different radial cutoff frequencies. These have unit height and take the position of $\mathrm{H}\left(\mathrm{f}_{\mathrm{x}}, \mathrm{f}_{\mathrm{y}}\right)$ in Eq. [A3]. The shaded (dark) area shows the passband region for a given filter. The respective upper radial frequency cutoffs are $1,1 / 2$, and $1 / 4$ expressed as fractions of the highest frequency component (i.e. $\mathrm{f}_{\mathrm{c}}$ ). These are lowpass filters. Frequencies at or below the cutoff are passed (without modification) and frequencies above the cutoff (white areas in Figure 2) are blocked totally. The bottom row of Figure 2 shows the respective filter outputs. From left to right, the outputs become grainy and then more-coarse corresponding with the cutoff frequency reduction. The effect of the filtering can be assessed by considering the amount of the frequency plane that is not blocked (the fractional passband). The percentage of the of the Fourier plane covered by each filter is given by: $\pi / 4 \times(\text { the pass band fraction })^{2} \times 100 \%$. The first filter passes all radial frequencies from zero to $\mathrm{f}=\mathrm{f}_{\mathrm{c}}$, which is the majority of the frequency plane (about $79 \%$ ). Therefore, the output resembles the input image of noise, slightly blurred. The top row of Figure 3 shows three additional filters with these cutoff frequency fractions: $1 / 8,1 / 16$, and $1 / 32$. The bottom row of Figure 3 shows three respective outputs. From left to right, the coarse quality assumes a lumpy and more long-range structural characteristic. This emerges because the remaining part of the spectrum is constrained to lower spatial frequencies corresponding to texture with longer-range spatial periodicities. For example, the last filter passes approximately $0.077 \%$ of the frequency plane. 


\subsection{Disk Filter Spatial Kernels}

The corresponding spatial kernels for the disk filters are described in this section. The general expression (Eq. [A9] - Eq. [A15]) for these kernels is given by

$$
h_{a}(x, y)=h_{a}(r)=a \frac{J_{1}(2 \pi a r)}{r},
$$

where $J_{1}$ is first order Bessel function, $r=\sqrt{x^{2}+y^{2}}, 0 \leq a \leq f_{c}$, the radial scaling parameter, a, was introduced as an index for $\mathrm{h}(\mathrm{x}, \mathrm{y}), \mathrm{d}=0.07 \mathrm{~mm}$, and $\mathrm{f}_{\mathrm{c}} \approx 7.14$ cycles $/ \mathrm{mm}$. A similar FT relationship is encountered in Fraunhofer diffraction for a circular aperture, resulting in Airy bands [32]. These kernels were also generated numerically by inverting the disk filters with the discrete FT. Letting $a=f_{c}, f_{c} / 2$, and $\mathrm{f}_{c} / 4$ gives the kernels shown in Figure 4. Theoretical plots (sold curves) are compared with the numerically derived kernels (dots); the kernels for the filters shown in Figure 3 follow the same trend and are not shown. A lowpass filter can be considered as a weighted averaging (blurring) process. The spatial extent of the averaging for these kernels can be crudely approximated by the width of the kernel's central lobe; summations over the more rapidly oscillating part of these kernels tend to cancel. For example, the widths (w) of the central lobes of the kernels shown in Figure 4 from left to right are approximately $\mathrm{w}=0.25 \mathrm{~mm}$ (about 4 pixels), $0.5 \mathrm{~mm}$ and $1.0 \mathrm{~mm}$. The extent of the area averaging is approximately $\pi \times \mathrm{w}^{2}$. Similar estimates can be derived by considering the spatial period of the average frequency

passed by a given filter: (i.e. $\left.\frac{(a+0)^{-1}}{2}\right) \approx \mathrm{w}=0.28 \mathrm{~mm}, 0.55 \mathrm{~mm}$ and $1.1 \mathrm{~mm}$, respectively. These estimates describe the spatial extent of the lumpiness shown in these illustrations, in agreement with the central lobe estimations.

\subsection{Fourier Radial Band Analysis}

The radial band analysis and its relationship to spatial domain filtering are described in this section. The general theoretical expressions for the image domain kernels are developed and compared with kernels derived numerically from Fourier inversion. Texture captured by specific bands are also illustrated.

The power spectrum is divided into a set of $\mathrm{n}$ concentric bands, where $\mathrm{n}$ is a variable adjustable integer parameter. The summation over each band produces $n$ measures. The radial bandwidth is given by $\Delta=f_{c} / n$. Each band is indexed with $m$ for $m=0$ to $n-1$. The center of the $m^{\text {th }}$ band is given by $(m+1 / 2) \times \Delta$, and the sum is taken over these limits: $(\mathrm{m} \Delta,(\mathrm{m}+1) \Delta]$ for the $\mathrm{m}^{\text {th }}$ measure; for presentation purposes, a given sum was taken over the entire radial band (complete ring), which is not necessary to capture the portion of the variance contained in the respective band due to spectral symmetry (discussed in Section 3.5 in more detail). Figure 5 shows the band-architecture in the FD using a coarse illustration with $\mathrm{n}=10$ giving $\Delta=\mathrm{f}_{\mathrm{c}} / 10$. The exterior regions not captured by the bands are referred to as the corners and can be summarized as an additional measure; for reference 
in total, this is a high-pass filter. Two related measures can be derived from the radial bands. One measure is the summarized power in a given band referenced as $\mathrm{P}_{\mathrm{m}}$. The other measure, $\mathrm{p}_{\mathrm{m}}$, is normalized at the image level given by $\mathrm{p}_{\mathrm{m}}=\mathrm{P}_{\mathrm{m}} /$ (total power from all bands + the power in the corners or equivalently the total power). In practice and depending on the analysis, the $\mathrm{m}=0$ component can be left out in the normalization and the analysis. This normalized measure may be used to compare images with different intensity scales. In this report, we focus on $\mathrm{P}_{\mathrm{m}}$. As illustration, this analysis was applied to the peppers image shown in Figure 6 (left) with $\mathrm{n}=41$, noting $\Delta$ is not important in this illustration. $\mathrm{P}_{\mathrm{m}}$ is shown in right side of Figure 6, which is easily visualized as a onedimensional radial spatial frequency plot. Similarly, the band analysis can also be used to compare spectra by considering variations in the respective $\mathrm{P}_{\mathrm{m}}$ ( or $\mathrm{p}_{\mathrm{m}}$ ) as illustrated previously [22, 27].

Band locations in Cartesian spatial frequency coordinates are illustrated to facilitate the description of radial spatial frequencies in the image domain provided in the next section. The support region in the frequency domain for a given band can also be described by considering discrete values for the radial frequency variable, $f$ $=\mathrm{m} \times \Delta$, and projecting it into Cartesian frequency coordinates. The measure from the $\mathrm{m}^{\text {th }}$ band is derived from the summing power spectrum over its respective area between the coordinate points, $\left(f_{x}, f_{y}\right)$, defining the interior radial border expressed as

$$
\mathrm{f}_{\mathrm{x}}=\mathrm{m} \times \Delta \times \cos (\phi) \text { and } \mathrm{f}_{\mathrm{y}}=\mathrm{m} \times \Delta \times \sin (\phi),
$$

and the coordinates defining the outer radial border

$$
\mathrm{f}_{\mathrm{x}}=(\mathrm{m}+1) \times \Delta \times \cos (\phi) \text { and } \mathrm{f}_{\mathrm{y}}=(\mathrm{m}+1) \times \Delta \times \sin (\phi)
$$

with

$$
\tan (\phi)=\frac{f_{y}}{f_{x}} .
$$

Radial spatial frequencies are illustrated in the spatial domain. In these examples, bandwidths corresponding with our recent work in mammography were used for illustration purposes (i.e. $\mathrm{n}=86$ and $\mathrm{f}_{\mathrm{c}} \approx$ $7.14 \mathrm{cc} / \mathrm{mm}$ ). For $\mathrm{m}=1$, f ranges from $\Delta$ to $2 \times \Delta$ or from 0.083 to 0.166 cycles $/ \mathrm{mm}$ (radially). Figure 7 shows $\mathrm{f}$ from the origin to two points on the inner band boundary in the Fourier plane; these points are defined as $f=f_{1}$ and $\mathrm{f}=\mathrm{f}_{2}$ for these angles: $\phi_{1}=\pi / 6$ and $\phi_{2}=\pi / 3$, or 30 degrees and 60 degrees, respectively. Using Eq. [5] gives: $\mathrm{f}_{\mathrm{x}} \approx 0.072$ and $\mathrm{f}_{\mathrm{y}} \approx 0.041$ for $\mathrm{f}_{1}$; and $\mathrm{f}_{\mathrm{x}} \approx 0.041$ and $\mathrm{f}_{\mathrm{y}} \approx 0.072$. Figure 8 (top-middle) shows the image expressed as

$$
C_{1}=\cos [2 \pi(0.072 x+0.041 y)]
$$


This is a corrugation [29, 33], shown in Figure 8 (top-left) with a peak to peak distance of $\frac{1}{\mathrm{f}}=\frac{1}{\Delta} \approx 12 \mathrm{~mm}$ (i.e. spatial period) measured along any line inclined relative to the $\mathrm{x}$-axis by $\pi / 6$. The superimposed red line is one such example. The image, shown in Figure 8 (bottom-left), corresponding to $\mathrm{f}_{2}$ is given by

$$
\mathrm{C}_{2}=\cos [2 \pi(0.042 \mathrm{x}+0.072 \mathrm{y})] \text {. }
$$

This is another corrugation with the same radial spatial frequency and period as $\mathrm{C}_{1}$ measured along any line inclined relative to the $\mathrm{x}$-axis by $\pi / 3$. Any point on the inner boundary in the FD produces a corrugation in the image domain with the same radial frequency, but in different directions. Similarly, we extend each line along the same directions to the outer boundary given by $\mathrm{f}=2 \times \Delta$, defining the $\mathrm{f}_{3}$ and $\mathrm{f}_{4}$ points shown in Figure 7 . These produce two more corrugation images: $\mathrm{C}_{3}$ (Figure 8 top-middle) and $\mathrm{C}_{4}$ (Figure 8 bottom-middle) with

peak to peak distances $\frac{1}{2 \times \Delta} \approx 6 \mathrm{~mm}$ measured along the respective directions. Profiles through these corrugations are shown in Figure 8 (top-right) with the same color-coding. Profiles through the corrugations in the bottom row are also shown in Figure 8 (bottom-right) using the same color coding. Thus, the image texture captured between the inner and boundaries viewed in the image domain is a sum of two-dimensional corrugations with spatial frequencies ranging between $0.083-0.166$ cycles $/ \mathrm{mm}$ of varying amplitudes for $\theta$ ranging from $0-2 \pi$. It follows, image structure captured within this radial band has spatial periodicities on the order of $6-12 \mathrm{~mm}$. As illustrated, a given corrugation in a radial system (image domain) has a defined direction. The unit vector in the spatial domain that points in the direction of a given corrugation is given by

$$
\mathbf{d}=\frac{\mathrm{f}_{\mathrm{x}} \mathbf{i}+\mathrm{f}_{\mathrm{y}} \mathbf{j}}{\sqrt{\mathrm{f}_{\mathrm{x}}^{2}+\mathrm{f}_{\mathrm{y}}^{2}}}
$$

where $\mathbf{i}$ and $\mathbf{j}$ are unit vectors along $\mathrm{x}$ and $\mathrm{y}$ directions, respectively. For example, $\mathbf{d}$ points in the direction of the red and blue lines superimposed on Figure 8 (left and middle columns).

\subsection{Bandpass Filter Kernels}

The filter kernel (spatial domain) for a band with an arbitrary radial bandwidth is derived from the disk filter expression. Letting $a_{2}>a_{1}$ with $a_{2} \leq f_{c}$, the kernel for the radial band bordered by $f=a_{2}$ on the exterior and $f=a_{1}$ on the interior can be derived by considering the radial integration expressed in Eqs. [A11-A14] from the origin to $\mathrm{a}_{1}$, from the origin to $\mathrm{a}_{2}$, and then taking the difference giving

$$
h_{b}(r)=h\left(r, a_{2}\right)-h\left(r, a_{1}\right)=a_{2} \frac{J_{1}\left(2 \pi a_{2} r\right)}{r}-a_{1} \frac{J_{1}\left(2 \pi a_{1} r\right)}{r},
$$

where the subscript, $b$, indicates bandpass filter and $a_{2}-a_{1}$ gives the corresponding pass bandwidth. A similar expression is also encountered in Fraunhofer diffraction for a centrally obscured aperture [32]. Equation [9] shows 
the output of the filtering process can be interpreted as the difference between two spatial scales; the output image is equivalent to differencing two lowpass filtered versions of the input image. In this and the related developments, these limits were used: $\left[a_{1}, a_{2}\right]$.

Two example profiles are used to compare theoretical expressions from Eq. [9] with numerically derived filter profiles. In the first example, in Figure 9 (top), shows both the band in the FD from the previous mammography example (i.e. $\mathrm{m}=1$ ) with $\mathrm{a}_{1}=\Delta$ and $\mathrm{a}_{2}=2 \times \Delta$, and the corresponding spatial domain texture that it produces (or captures) in the bottom pane. The corresponding theoretical kernel (solid) is compared with the numerically derived kernel (dots) in Figure 10 (left). The center lobe of this kernel spans about 10mm, giving a good estimate of the spatial structure captured by this filter. This also agrees with the estimate made with the spatial periods from the boundaries above. In the second example, Eq. [9] is applied more generally as a bandpass filter, bypassing the filter bank description. Using the parameters from our mammography work, the bandwidth was defined with $\mathrm{a}_{1}=25 \times \Delta$ and $\mathrm{a}_{2}=39 \times \Delta$ (approximately $0.30 \times \mathrm{f}_{\mathrm{c}}$ and $0.45 \times \mathrm{f}_{\mathrm{c}}$, respectively). Figure 10 (right) shows the corresponding kernel's theoretical profile (solid) compared with the numerically derived profile (dots). The band in the FD is shown in Figure 11 (top-left) and the corresponding texture is shown in Figure 11 (bottomleft). The spatial scale of the texture captured by this filter is on the order of $\frac{1}{a_{1}}$ to $\frac{1}{a_{2}}$ or $0.30 \mathrm{~mm}$ to $0.48 \mathrm{~mm}$; this also corresponds with the central lobe width of its kernel shown in Figure 10 (right-pane), which is roughly $0.4 \mathrm{~mm}$.

\subsection{Radial Bands and Directionality}

As a first illustration of capturing directionality, we use the corrugations from the previous example. Summing the four corrugations with even weights produces a composite image expressed as

$$
\mathrm{C}=\mathrm{C}_{1}+\mathrm{C}_{2}+\mathrm{C}_{3}+\mathrm{C}_{4}
$$

shown in Figure 12. The pronounced striations are in the diagonal direction because the sum was restricted to components with Fourier angles in the first quadrant. This illustrates there is a relationship between the orientation of linear structure and the direction of the related corrugations. The relationship (an idealization) between $\phi$ in FD and the directional angle, $\beta$, relative to the $x$-axis in the image domain is given by $\beta=\frac{\pi}{2}-\phi$, where $\beta$ is illustrated in Figure 8 (top-right, coded green). As $\beta$ approaches $\frac{\pi}{2}$ (linear structure's orientation approaches the $\mathrm{y}$-direction), the corrugation pointes along the $\mathrm{x}$-direction, $\mathbf{d}=\mathbf{i}$, from Eq. [8]. On the other extreme, as $\beta$ approaches 0 (linear structure's orientation is in the $\mathrm{x}$-direction), the corrugation points along the $\mathrm{y}$-direction, $\mathbf{d}=$ 
j. Thus, the orientation of linear structure in $C$ is expected to lie within the range from $\beta=\frac{\pi}{6}$ to $\beta=\frac{\pi}{3}$ (i.e. between 30-60 degrees).

Following the previous illustration, the approach was extended to capture texture with directional attributes by considering bands restricted to radial arcs in the FD. We introduce this additional angular dimension with specific examples, which is defined more precisely below. First, the wider bandpass filter (Figure 11, topleft) was modified by restricting it to a specific arc illustrated in Figure 12 (top-middle), where the radial boundaries are $a_{1}$ and $a_{2}$ from the previous example. In the upper right quadrant relative to the $f_{x}$ axis, the lower radial boundary is inclined at $\frac{\pi}{6}$ and the upper at $\frac{\pi}{3}$ giving the central angular band location of $\phi_{\mathrm{c}}=\frac{\pi}{4}$. Figure 12 (bottom-middle) shows the corresponding linear structure created by this filter when applied to noise. The linear striations are oriented in a direction centered about the angle, $\beta_{\mathrm{c}}=\frac{\pi}{2}-\frac{\pi}{4}$, relative to the $\mathrm{x}$-axis (see Figure 8, topright for $\beta$ ). Figure 12 (top-right) shows the same arc with $\mathrm{f}$ extended to $\mathrm{f}_{\mathrm{c}}$. The corresponding linear structure is illustrated in Figure 12 (bottom-right); the more resolved striations are due to extending the radial bandwidth in comparison with the previous example (bottom-middle). These plots also show examples of spectral symmetry for the first quadrant. In sum, these filters have both radial and angular (i.e. texture and directionality in the image domain) bandwidths. The angular band can be adjusted to capture linear structure for arbitrary orientations while the radial band can be adjusted for varying spatial scales.

The peppers image (Figure 6) was processed with the filters shown in Figure 12 to illustrate a practical application. The three respective outputs are shown in Figure 13. Outputs were post-processed for viewing purposes by taking the absolute value and over contrasting. The spatial scale captured by the bandpass filter (Figure 13, left pane) is noted by the structure that is most prominent. The middle and right panes in this figure show the outputs from the filters with angular preferences. The pronounced linear structure passed by the angularrestricted filters is centered about $\beta_{\mathrm{c}}=\frac{\pi}{4}$. As in the previous example, the structure shown in the top-right pane is more resolved relative the structure in the middle-pane due to the increased radial bandwidth of the respective filter (Figure 12, right-pane).

The FD indexing was formally extended to include directionality paralleling the band analysis in Section 3.3. Including the second index gives $\mathrm{P}_{\mathrm{ml}}$, where 1 references angular FD dependency. In addition to the $\mathrm{n}$ radial bands, the spectrum was divided into $\mathrm{k}$ angular bands (arcs) indexed by $1=0$, through $\mathrm{k}-1$ with angular bandwidths given by $\phi_{\mathrm{k}}=\frac{\pi}{\mathrm{k}}$. The angular limits for each arc are given by, $\left[1 \times \phi_{\mathrm{k}},(1+1) \times \phi_{\mathrm{k}}\right)$, and the central location by, $\left(1+\frac{1}{2}\right) \times \phi_{\mathrm{k}}$. The spectrum is summarized with $\mathrm{n} \times \mathrm{k}$ measures, in indexed to specific textures in the image domain, in addition to the measurement from the corners. Figure 14 shows a coarse illustration of dividing the 
Fourier plane with $\mathrm{n}=5$ and $\mathrm{k}=6$, giving 31 measurements. Angular increments are measured from the positive $\mathrm{f}_{\mathrm{x}}$ axis in a counterclockwise rotation. Although the entire plane is shown in Figure 14 and related examples, the analysis was restricted $[0, \pi]$ without loss of generality by considering spectral symmetry: $S\left(f_{x}, f_{y}\right)=S\left(-f_{x},-f_{y}\right)$; and $S\left(-f_{x}, f_{y}\right)=S\left(f_{x},-f_{y}\right)$. As illustration, intra-image comparisons were evaluated using the peppers image (Figure 6) and CWA image shown in Figure 15 with $n=41$ and $k=6 . P_{m l}$ is plotted as a function of 1 , giving six radial plots for each image, shown in Figure 16 (peppers in left-pane and CWA in the right-pane). These plots are shown on a logarithmic scale and color coded for these arcs: $\left[0, \frac{\pi}{6}\right),\left[\frac{\pi}{6}, \frac{\pi}{3}\right),\left[\frac{\pi}{3}, \frac{\pi}{2}\right),\left[\frac{\pi}{2}, \frac{2 \pi}{3}\right)\left[\frac{2 \pi}{3}, \frac{5 \pi}{6}\right),\left[\frac{5 \pi}{6}, \pi\right)$ corresponding with $\mathrm{P}_{\mathrm{m} 1}$ (black), $\mathrm{P}_{\mathrm{m} 2}$ (blue), $\mathrm{P}_{\mathrm{m} 3}$ (red), $\mathrm{P}_{\mathrm{m} 4}$ (green), $\mathrm{P}_{\mathrm{m} 5}$ (pink), and, $\mathrm{P}_{\mathrm{m} 6}$ (cyan), respectively. For the peppers image (Figure 15, left), these plots indicate the spectrum does not exhibit strong angular (directional) characteristics (i.e. the image does not have pronounced linear structure in preferred directions) because there are relatively minor deviations at a given $\mathrm{m}$. In contrast, the CWA image (Figure 15, right) shows strong deviation that can be explained by considering its pronounced linear structure in well-defined directions. The radial line from the origin superimposed on this image intersects pronounced linear structure at $\frac{\pi}{2}$ (90 degrees): $\phi \approx 0.11 \times \pi$ (approximately, 20 degrees), giving $\beta \approx 0.38 \pi$ (70 degrees). As illustrated in Figure 16 (right), the plots corresponding to the first angular interval (black, $0-\frac{\pi}{6}$ or 0-30 degrees) and last angular interval (cyan, $\frac{5 \pi}{6}-\pi$ or $150-180$ degrees) deviate from the other angular intervals plots for $\mathrm{m} \leq 10$ and are in close agreement at each $\mathrm{m}$. These two plots essentially track each other due to the symmetry in the image across the y-axis with respect to the pronounced linear structure.

To illustrate the two-dimensional indexing and its relationship to spatial scale and orientation, the CWA was filtered with wider radial bandwidths in tandem with the same angular bands (same color coding). The extended bands were based on the CWA analysis in the previous example (same radial and angular divisions). Seven radial bandpass filters were constructed by combining these bands: (1) $\mathrm{m}=5-10$; (2) $\mathrm{m}=10-15$; (3) $\mathrm{m}$ $=15-20 ;(4) \mathrm{m}=20-25 ;(5) \mathrm{m}=25-30 ;(6) \mathrm{m}=30-35$; and (7) $\mathrm{m}=35-40$. We show the six angular bands for each wider radial band in one illustration. For each illustration for viewing purposes, the $95^{\text {th }}$ percentile for the combined cumulative pixel value (absolute value) distribution for each filtered image was estimated by integrating the normalized histogram. Pixel values at or above this cut point were set to the maximum value in the respective illustration. These illustrations are shown in figures 17-23. The structure illustrated in Figure 15 is prominent in Figure 17 and Figure 18 (bands 5-10 and 10-15) in the black and cyan angular bands. When following these angular bands, this structure is slightly faded in Figure 19 (bands 15-20) and then transitions to chatter in following images. In the next example, we focus on the floor that appears to have tile with grout lines running into the plane of the figure (our focus) and across the plane. The lines into the plane are visible in these bands: 
(1) in the black and cyan bands for the tile in the central part of the image that intersect the $x$-direction with $\beta \approx$ $\frac{\pi}{2}$ or $\phi \approx 0$; or (2) in the adjacent blue and pink angular bands away from the central part of the image; these are most prominent in the $2-4^{\text {th }}$ wider radial bands (Figure 19, Figure 20, and Figure 21). The lines change direction in outer parts of the image due to photographic influences. For the outer lines on the right-side, we estimate $\beta \approx$ $0.21 \pi$ (about 38 degrees) giving $\phi \approx 0.29 \pi$ (about 52 degrees). Thus, the angular cutoffs are clearly indicated in the shift from the cyan/black bands to the blue/pink bands at the respective scales.

\subsection{Spectral Modeling}

The band analysis can be used to evaluate the fidelity of spectral models under certain conditions [22] using the peppers image. Although not necessary, we assume radial symmetry (i.e. $P_{m}$ is used) and let $S_{m}$ (f) define the spectral model. The model can be fit to a one-dimensional function by integrating over bands

$$
\mathrm{R}(\mathrm{m})=\mathrm{c} \int_{\Delta \mathrm{m}}^{\Delta(\mathrm{m}+1)} \mathrm{S}_{\mathrm{m}}(\mathrm{f}) \mathrm{fdf},
$$

where $\mathrm{c}$ is an adjustable constant. We assume the following model for the peppers image

$$
\mathrm{S}_{\mathrm{m}}(\mathrm{f})=\frac{1}{\left[\mathrm{f}_{0}^{2}+\mathrm{f}^{2}\right]^{\alpha}}
$$

where $\mathrm{f}_{0}$ and $\alpha$ are adjustable parameters determined from the curve fitting analysis. Integration over adjacent bands gives

$$
\mathrm{R}(\mathrm{m})=2 \pi \int_{\Delta \mathrm{m}}^{\Delta(\mathrm{m}+1)} \frac{\mathrm{fdf}}{\left[\mathrm{f}_{0}^{2}+\mathrm{f}^{2}\right]^{\alpha}}=\mathrm{c}\left[\frac{1}{\left[\mathrm{q}^{2}+\mathrm{m}^{2}\right]^{\alpha-1}}-\frac{1}{\left[\mathrm{q}^{2}+(\mathrm{m}+1)^{2}\right]^{\alpha-1}}\right],
$$

where $\mathrm{q}$ is a lumped constant to be determined and $\mathrm{c}$ is a lumped scaling factor this is not important. Note for small q, this spectral model approximates a power law. The theoretical fitted curve (sold) is compared with numerical curve (dots) in Figure 6 (right) with $\alpha \approx 1.75$ and $\mathrm{q}^{2}<10^{-13}$. Thus, a good approximation, the spectrum for the peppers image obeys an inverse power law expressed as $\mathrm{S}(\mathrm{f}) \sim \mathrm{f}^{-2 \alpha}$.

\subsection{Improvements}

If the image domain approach is important, the spatial response for the radial band filters can be improved. One approach is to remove the steep (infinite) filter profiles and broaden the bandwidths. We define the center of a given band as $\mathrm{m}_{\mathrm{c}}=(\mathrm{m}+1 / 2) \times \Delta$ and define the modified Fourier domain filter as

$$
\mathrm{H}(\mathrm{f})=\exp -\frac{\left(\mathrm{f}-\mathrm{m}_{\mathrm{c}}\right)^{2}}{2 \sigma^{2}}
$$

where $\sigma$ can be defined in terms of $\Delta$. The width that gives $H(f)=1 / 2$ for $f=m_{c} \pm k \times \Delta$ is given by 


$$
\sigma=\frac{\mathrm{K} \times \Delta}{\sqrt{2 \ln (2)}}
$$

where $\mathrm{K}$ is a positive integer. Figure 16 shows the profiles for a band filter and the improvements for $\mathrm{m}=30$ and $\mathrm{K}=2$ in the left pane. The spatial domain kernels are compared in the right pane. Note that the modified kernel tappers to zero more rapidly than the unmodified kernel. For completeness following Eq. [A4], the spectral density for this modification is expressed as

$$
\mathrm{S}(\mathrm{f})=\exp -\frac{\left(\mathrm{f}-\mathrm{m}_{\mathrm{c}}\right)^{2}}{\sigma^{2}}
$$

This approach can be extended to include angular bandwidths as well. In conjunction with modifying the filters, zero padding in the image domain can be used to offset the periodic wrap-around effects, making the discrete Fourier convolution relationships match that of theoretical [28]. Similarly, kernels can be truncated when performing image domain operations.

\section{Discussion and Conclusion}

The relationship between image texture and the FD was illustrated. Examples were provided within the context of describing a technique to summarize a two-dimensional power spectrum. This is a data reduction technique that can be used to visualize the power spectrum of a given image and make various spectral comparisons, among other applications. The reduction technique gives a full accounting of the image variance. Performing the analysis in a polar coordinate system enables a simple indexing scheme that relates image texture and its directional orientation to specific regions in the FD that can be varied easily. The tradeoff for this simple indexing is that the associated spatial domain kernels have poor localization properties and the measures can bear strong correlation (although not assessed in this work). This is in contrast with wavelet expansion that produces a multiscale orthogonal representation; the price paid for orthogonality is the octave sectioning of the Fourier view of the expansion images [34] (see [35, 36] for other examples). The radial kernel expression was derived in closed form to show the spatial characteristics. The more general angular dependent expression was not derived (the subject of ongoing work) but was analyzed empirically Because our approach is based on the FD, it is more applicable for characterizing field effects in the image domain in contrast with detecting local behavior. That is, the approach is applicable when spatial positional invariance is not important. In sum and depending on the type of analysis, it may be beneficial to develop features from the FD without considering the image domain as illustrated. 


\section{Acknowledgments}

This work was supported by National Institutes of Health Grants: R01CA166269, R01CA177150, and U01CA200464.

\section{Disclosers}

The authors have nothing to disclose. 


\section{Appendices}

\section{A1. Fourier Background}

A brief background in Fourier analysis and filtering is provided [28, 33, 37]. The continuous two-dimensional Fourier transform (FT) of the image, $\mathrm{f}(\mathrm{x}, \mathrm{y})$, is expressed as

$$
F\left(f_{x}, f_{y}\right)=\int_{-\infty}^{\infty} \int_{-\infty}^{\infty} f(x, y) \exp -2 \pi i\left(f_{x} x+f_{y} y\right) d x d y
$$

where, $i$ is the unit imaginary, $x$ and $y$ are Cartesian spatial coordinates and $f_{x}$ and $f_{y}$ are Cartesian spatial frequency coordinates. Lower case indicates the spatial domain and upper case the Fourier domain. Spatial coordinates are measured in $\mathrm{mm}$ and spatial frequencies in cycles/mm. The Fourier inversion operation that recovers $f(x, y)$ is expressed as

$$
f(x, y)=\int_{-\infty}^{\infty} \int_{-\infty}^{\infty} F\left(f_{x}, f_{y}\right) \exp 2 \pi i\left(f_{x} x+f_{y} y\right) d f_{x} d f_{y} .
$$

Filtering in the Fourier domain is a multiplication expressed as

$$
F_{H}\left(f_{x}, f_{y}\right)=H\left(f_{x}, f_{y}\right) F\left(f_{x}, f_{y}\right)
$$

where $\mathrm{H}\left(\mathrm{f}_{\mathrm{x}}, \mathrm{f}_{\mathrm{y}}\right)$ is the FT of the filter function, $\mathrm{h}(\mathrm{x}, \mathrm{y})$. The subscript $\mathrm{H}$ was introduced to indicate that $\mathrm{F}\left(\mathrm{f}_{\mathrm{x}}, \mathrm{f}_{\mathrm{y}}\right)$ was modified by $H\left(f_{x}, f_{y}\right)$. Thus, the filter shapes the spectral properties of $f(x, y)$. The power spectral density (or power spectrum) of an arbitrary function $\mathrm{G}\left(\mathrm{f}_{\mathrm{x}}, \mathrm{f}_{\mathrm{y}}\right)$ is defined as

$$
S\left(f_{x}, f_{y}\right)=G\left(f_{x}, f_{x}\right) G^{*}\left(f_{x}, f_{y}\right)
$$

where the asterisk indicates complex conjugate. Using Eq. [A2], the inverse FT of Eq. [A3] gives the convolution (filtering) in spatial domain

$$
f_{h}(x, y)=\int_{-\infty}^{\infty} \int_{-\infty}^{\infty} f\left(x^{\prime}, y^{\prime}\right) h\left(x-x^{\prime}, y-y^{\prime}\right) d x^{\prime} d y^{\prime} .
$$

We use the discrete Fourier forms of Eq.[A1]- Eq.[A5] in this work [28]. The details of the discrete FT are not discussed in detail here beyond quasi-discrete illustration related to the image variance, provided next.

The complex exponential in Eq. [A1] has two components expressed as

$$
\cos \left[2 \pi\left(f_{x} x+f_{y} y\right)\right] \text { and } \sin \left[2 \pi\left(f_{x} x+f_{y} y\right)\right] \text {. }
$$

We apply Eq. [A1] for discrete spatial frequencies. The amplitude (decomposition) for the cosine component at one specific coordinate in the spatial frequency plane at $f_{x}=u$ and $f_{y}=v$ is given by 


$$
A_{r}(u, v)=\iint_{x, y}^{r e g i o n} f(x, y) \cos [2 \pi(u x+v y)] d x d y,
$$

where the integration is constrained to the image region. The integral for the sine term gives the corresponding amplitude for the imaginary component expressed as $A_{i}(u, v)$. The magnitude at $\left(f_{x}, f_{y}\right)$ can be considered as a similarity gauge between the image and the respective sinewave. It is important to note that every point in the Fourier domain represents a linear combination that included every point in the image domain. The power (Eq. $[\mathrm{A} 4])$ at $\left(\mathrm{f}_{\mathrm{x}}=\mathrm{u}, \mathrm{f}_{\mathrm{y}}=\mathrm{v}\right)$ is given by

$$
\left|A_{\mathrm{r}}\right|^{2}=\mathrm{A}_{\mathrm{r}}^{2}(\mathrm{u}, \mathrm{v})+\mathrm{A}_{\mathrm{i}}^{2}(\mathrm{u}, \mathrm{v}) \text {. }
$$

The sum over all $\left(f_{\mathrm{x}}, \mathrm{f}_{\mathrm{y}}\right)$ using Eq. [A8] is equivalent to calculating the variance in the image domain [excluding $(0,0)$ if the mean is not zero]. The same variance relationship applies to Eq.[A4] and Eq. [A5].

\section{A2. Kernel Derivation for the Disk Filters}

The inverse FT for and arbitrary filter, $\mathrm{H}\left(\mathrm{f}_{\mathrm{x}}, \mathrm{f}_{\mathrm{y}}\right)$, is given by

$$
h(x, y)=\int_{-\infty}^{\infty} \int_{-\infty}^{\infty} H\left(f_{x}, f_{y}\right) \exp 2 \pi i\left(f_{x} x+f_{y} y\right) d f_{x} d f_{y} .
$$

Because the disk filters are radially symmetric with constant radius (radius =a) and unit height, we switch to polar coordinates in both domains: $(x, y)$ maps to $(r, \theta)$ in the spatial domain and $\left(f_{x}, f_{y}\right)$ maps to $(f, \phi)$ in the frequency domain with $\mathrm{f}=\sqrt{\mathrm{f}_{\mathrm{x}}^{2}+\mathrm{f}_{\mathrm{y}}^{2}}$ and $\mathrm{r}=\sqrt{\mathrm{x}^{2}+\mathrm{y}^{2}}$, where both angles range from 0 to $2 \pi$. For completeness, we develop two well-known relationships [29, 37] often developed by starting with the FT in the image domain: (i) the connection between the two-dimensional FT and Hankel transform (HT) when there is radial symmetry, and (ii) using the HT to find the inverse FT of the Fourier domain disk filters (i.e. pillboxes). The argument of the exponential expression in Eq. [A9] can be considered as inner product of the vector $\mathbf{r}$ with components $\mathrm{x}=$ $r \times \cos (\theta)$ and $y=r \times \sin (\theta)$, and the vector $\mathbf{f}$ with components $f_{x}=f \times \cos (\phi)$ and $f_{y}=f \times \sin (\phi)$. First, we use the polar relationships in both domains to give an alternative expression for the exponential argument in Eq. [A9] given by

$$
\mathrm{f}_{\mathrm{x}} \mathrm{x}+\mathrm{f}_{\mathrm{y}} \mathrm{y}=\mathrm{rf}[\cos (\phi) \cos (\theta)+\sin (\phi) \sin (\theta)]=\mathrm{rf} \cos (\phi-\theta),
$$

derived from cosine and sine multiplication identities. As common practice, we let the vector $\mathbf{r}$ point along the $\theta$ $=0$ axis in the spatial domain, switch the integration to polar coordinates in the frequency domain, and substitute Eq. [A10] into Eq. [A9] giving

$$
h(r)=\int_{0}^{a} \int_{0}^{2 \pi} H(f) \exp 2 \pi i[r f \cos (\phi)] d \phi f d f=\int_{0}^{a} \int_{0}^{2 \pi} H(f) \cos [2 \pi r f \cos (\phi)] d \phi f d f,
$$


where the imaginary component was dropped due to symmetry and $0 \leq a \leq f_{c}$. The angular integration defines the zero order Bessel function. Thus, the inverse FT, reduces to the HT of H(f) expressed as

$$
h(r)=2 \pi \int_{0}^{a} H(f) J_{0}(2 \pi r f) f d f .
$$

Letting $H(f)=1$ and making a change of variable with $z=\frac{f}{a}$ gives

$$
h(r)=2 \pi a^{2} \int_{0}^{1} J_{0}(2 \pi r a z) z d z
$$

Using integration tables [38] (see page 707, Eq. [5]) produces the kernel expression

$$
h(r, a)=a \frac{J_{1}(2 \pi a r)}{r}
$$

where the filter kernel is a function of $r$ and the parameter, a, defined over $\left(0, \mathrm{f}_{\mathrm{r}}\right]$. Both the disk filters and band filterers have unit height with exact cutoffs and are real indicating that the amplitude of $H(f)$ is equvalnt to that of $S(f)=H(f) H^{*}(f)=1$, which is a special case. 


\section{Figures}

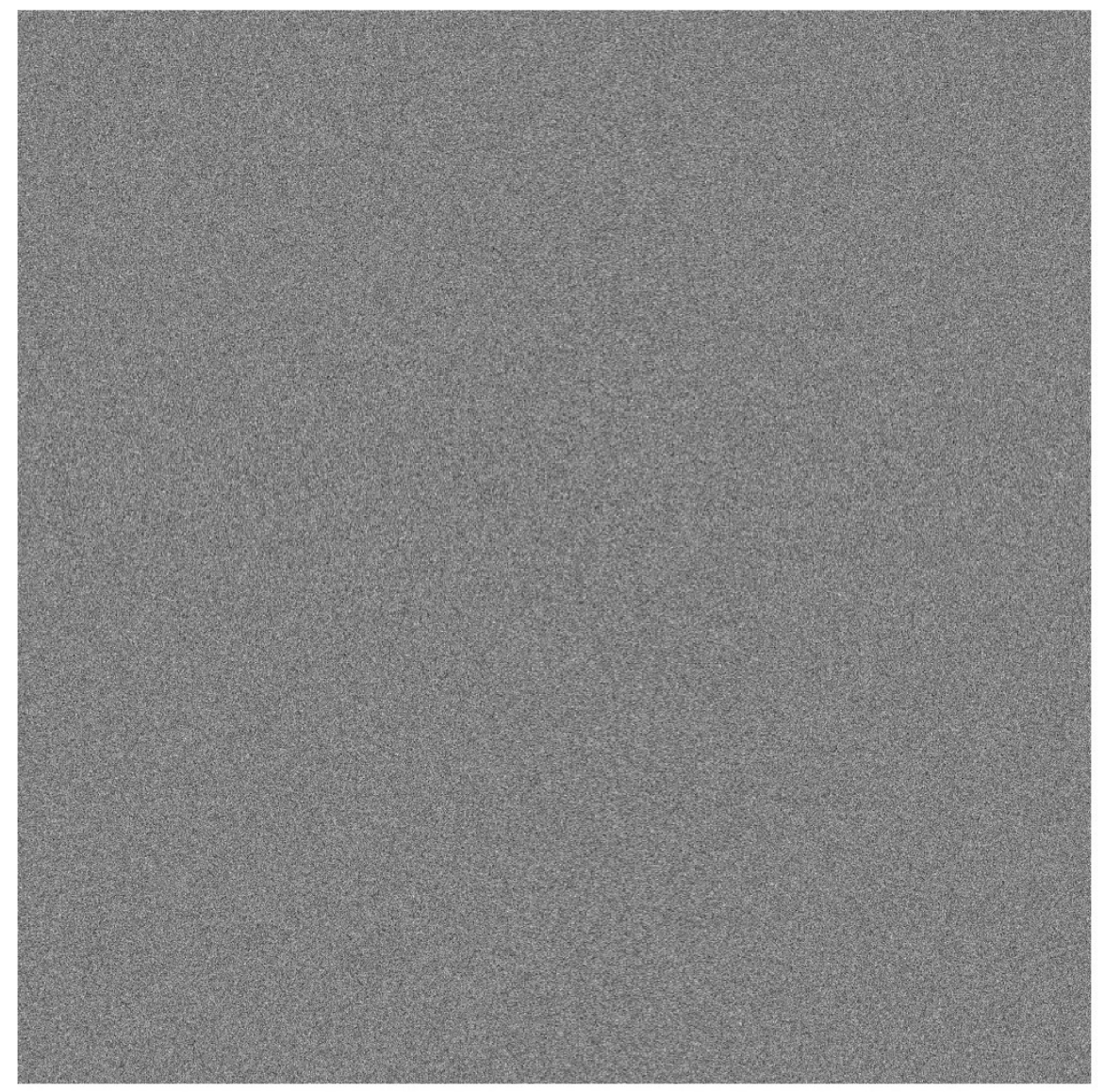

Figure 1: White Noise Illustration. This shows one realization of a random (white) noise image used as the inputs to the filtering processes. 

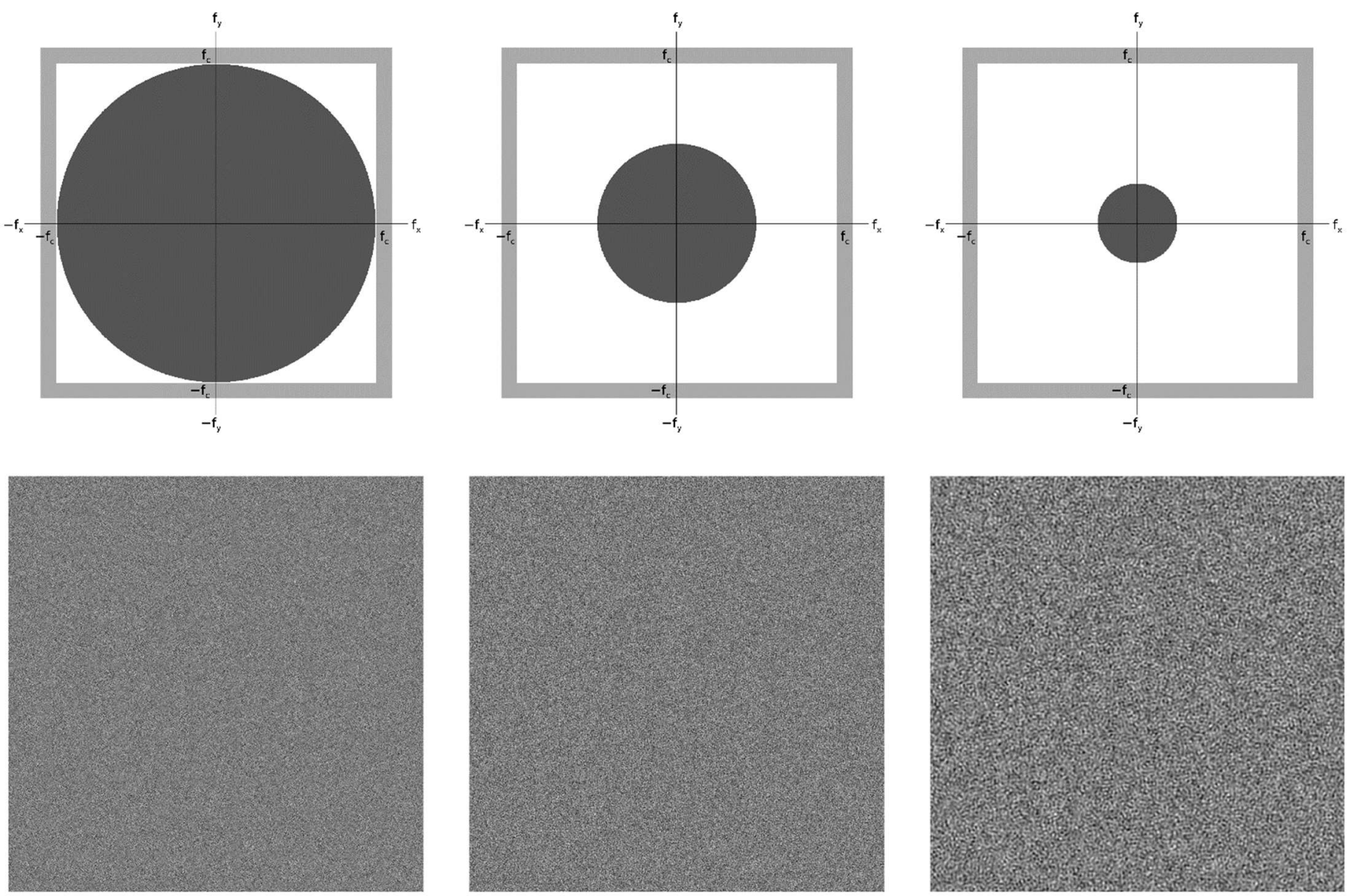

Figure 2: Filtering and Texture Illustration 1. The top row shows three disk filters in the Fourier domain with zero frequency in the middle. Filled circles show the passbands (dark area) for each filter (unit height). The larger rectangle (white area) defines the area corresponding to the band limited spatial frequency plane. The upper radial cutoff frequencies (i.e. disk radii) are 1, 1/2, and $1 / 4$ cited in fractions of the highest frequency component $\left(f_{c}\right)$ in Cartesian coordinates. White noise was filtered with each one of these filters. Filtered noise (bottom row) becomes coarser from left to right. The proportion of the of the Fourier domain area covered by each filter is $(\pi / 4) \times(\text { pass band fraction })^{2}$. 

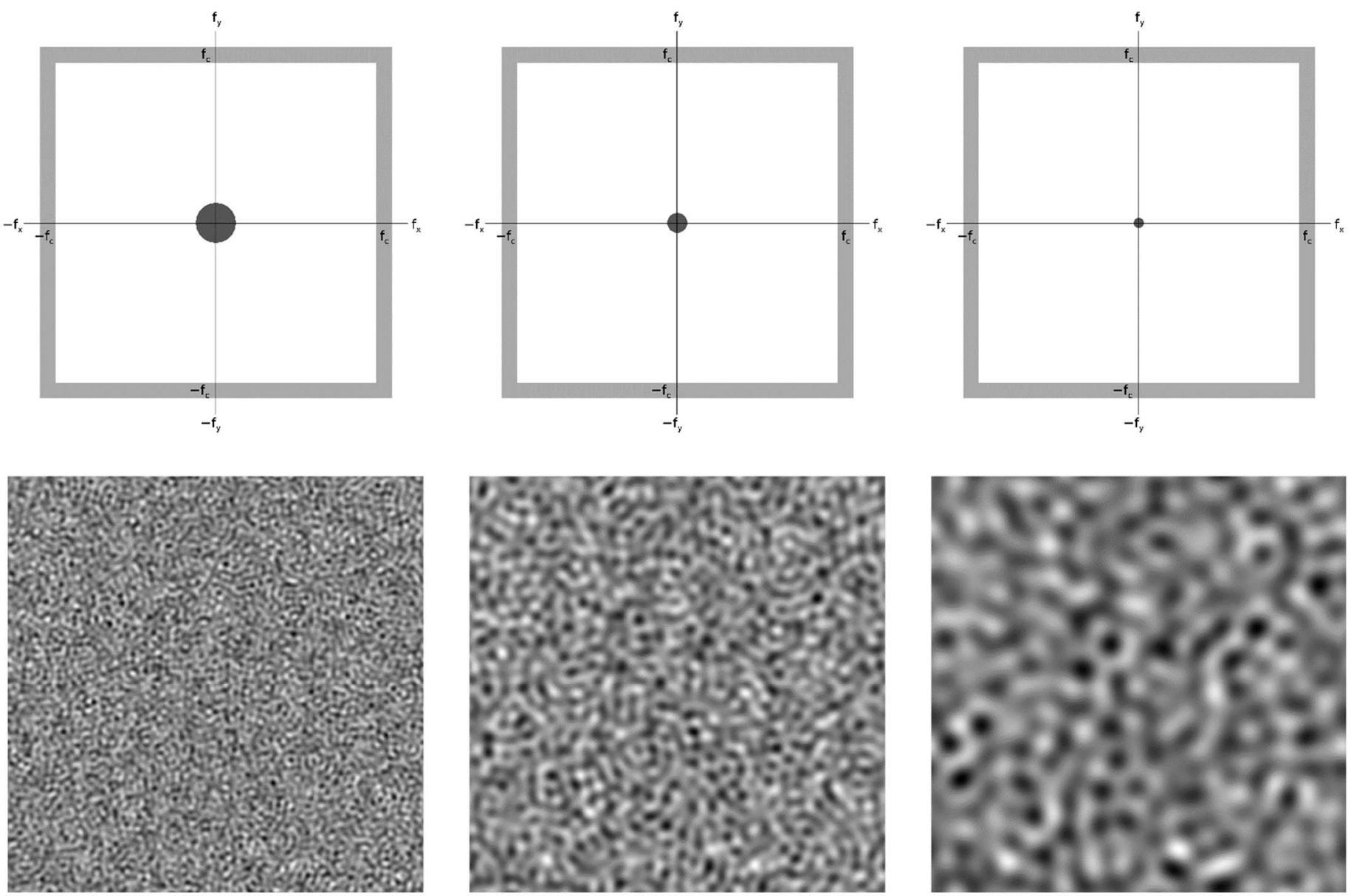

Figure 3: Filtering and Texture Illustration 2. The top row shows three disk filters in the Fourier domain with zero frequency in the middle. The filled circles show the passband (dark area) for each filter (unit height). The radial cutoff frequencies (i.e. disk radii) are 1/8,1/16, and 1/32 cited in fractions of the highest frequency component $\left(f_{c}\right)$ in Cartesian coordinates. White noise was filtered with each one of these filters. The filtered images (noise fields in the bottom row) become coarserfrom left to right and the textures begin to take on a more structural characteristic. The proportion of the of the Fourier domain area covered by each disk is $(\pi / 4) \times(\text { pass band fraction })^{2}$. 

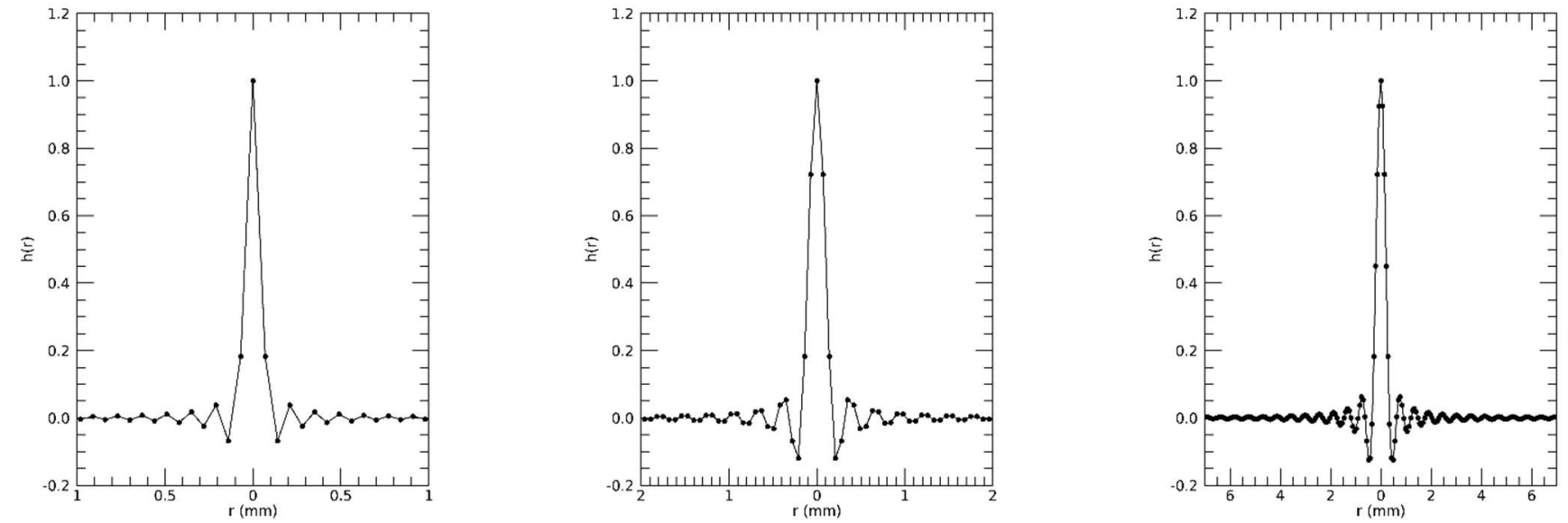

Figure 4: Disk Filter Kernel Radial Profiles. These plots show the filter kernels for the disk filters shown in Figure 2 as a function of $r$ with $a=f_{c}, f_{c} / 2$ and $f_{c} / 4$ (from left to right). The theoretical plots (solid) from Eq. (12) are compared with radial profiles (dots) from the Fourier transform of the disk filters (Figure 2). These are twodimensional non-separable radially symmetric kernels. All kernels were normalized to unity at the origin. 


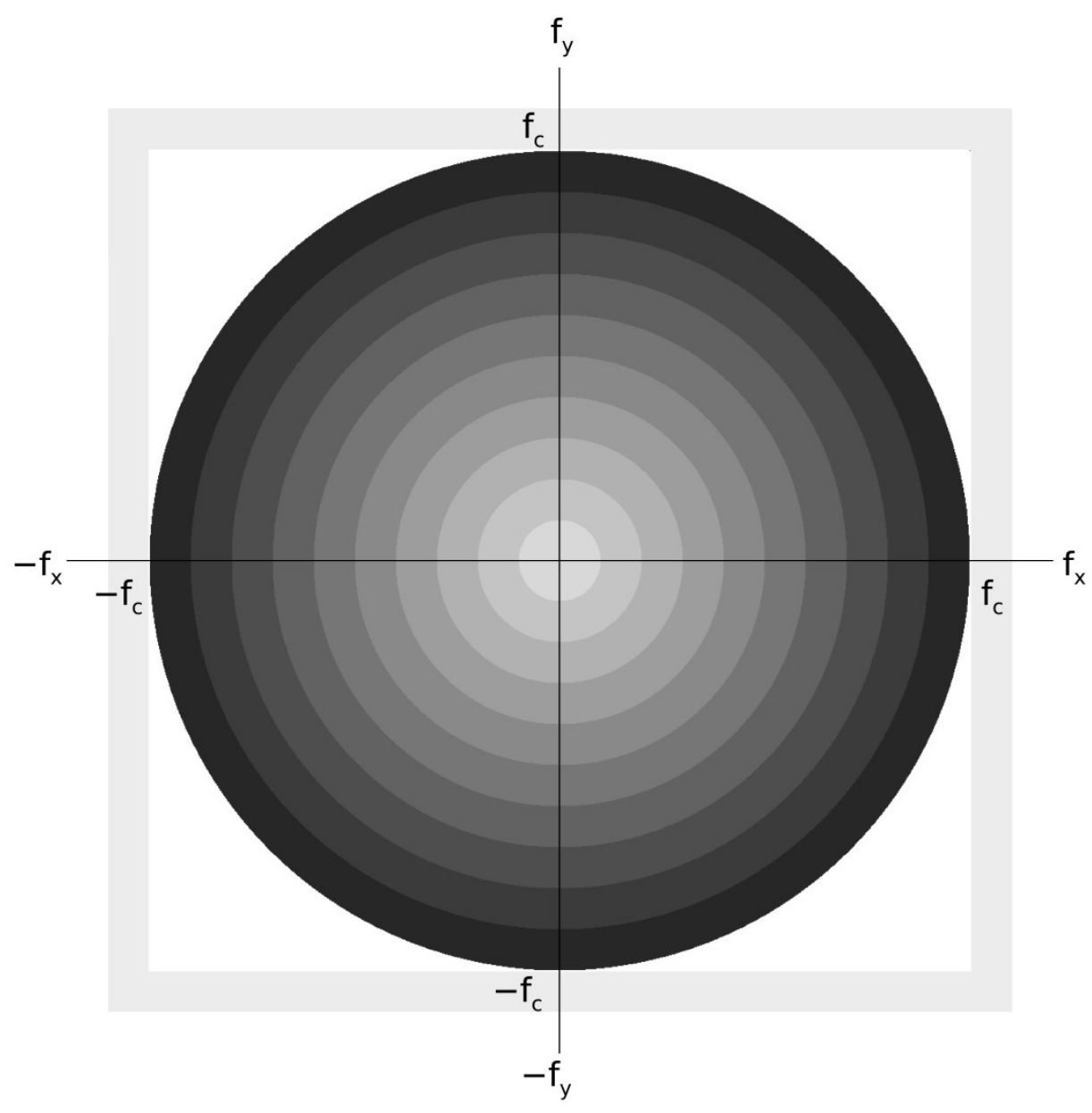

Figure 5: Fourier Radial Band Analysis Illustration. This shows the Fourier domain and the band architecture. In this coarse example, $\mathrm{n}=10$ bands for viewing purposes. The coordinates of the SE, NE, NW, and SW corners ($\left.f_{c}, f_{c}\right),\left(f_{c}, f_{c}\right),\left(-f_{c}, f_{c}\right)$ and $\left(-f_{c},-f_{c}\right)$, respectively, define the frequency plane. The zero frequency coordinate, $\left(f_{x}\right.$, $\left.f_{y}\right)=(0,0)$, is in the center of the plane. Bandwidths are $f_{c} / 10$ in the radial direction. In this numbering scheme, the center (white-disk) corresponds to $\mathrm{m}=0$ and the outer band with $\mathrm{m}=\mathrm{n}-1$, where $\mathrm{m}$ is the band index. The portion of the spectrum not covered by the disks is referred to as the corners enabling an additional measure. 

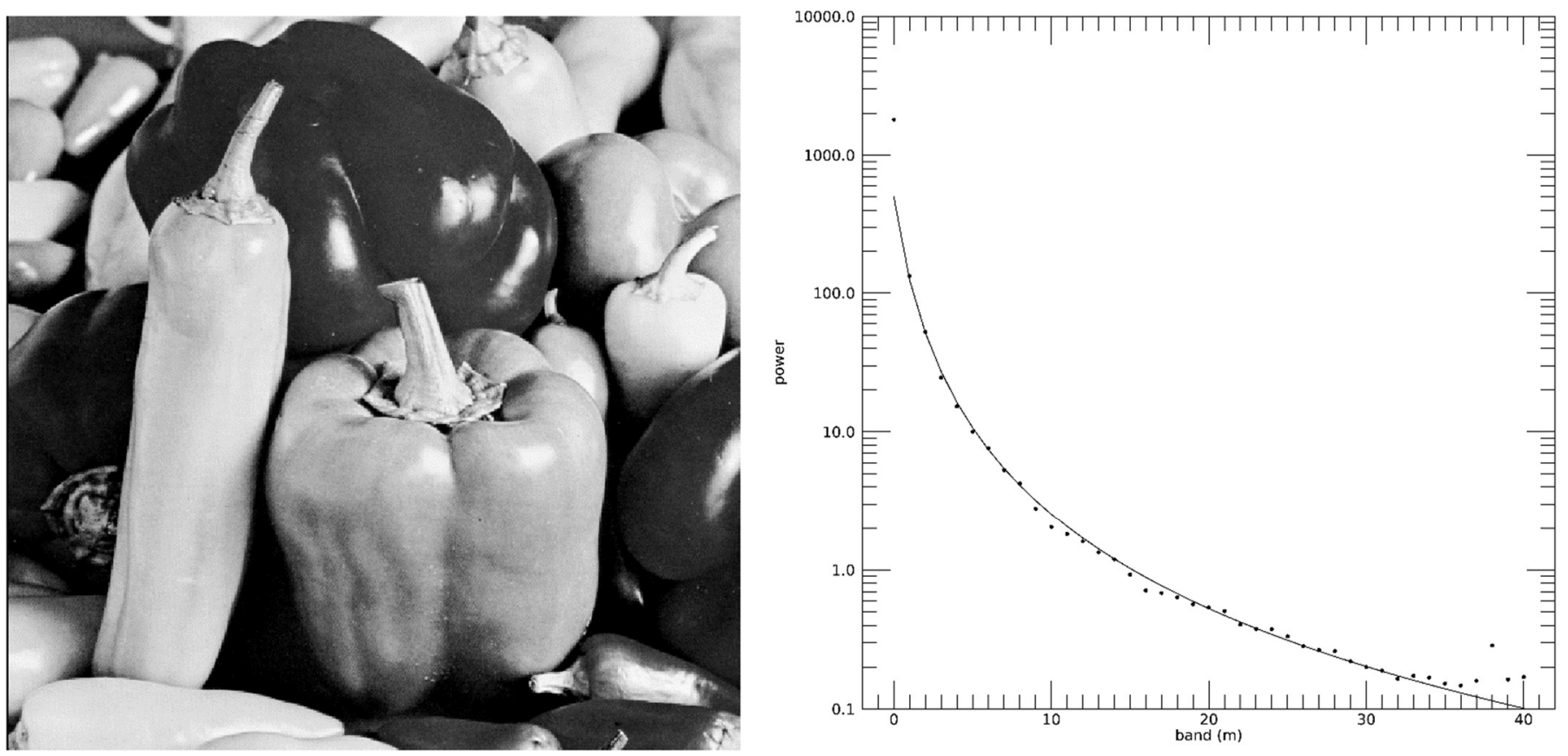

Figure 6: Radial Band Analysis of the Peppers Image. The peppers image is shown in the left pane: $511 \times 511$ pixels. The empirical radial band analysis (dots) for $\mathrm{n}=41$ is shown in the right pane. The solid line (right) shows the theoretical model from Eq. [12]. 

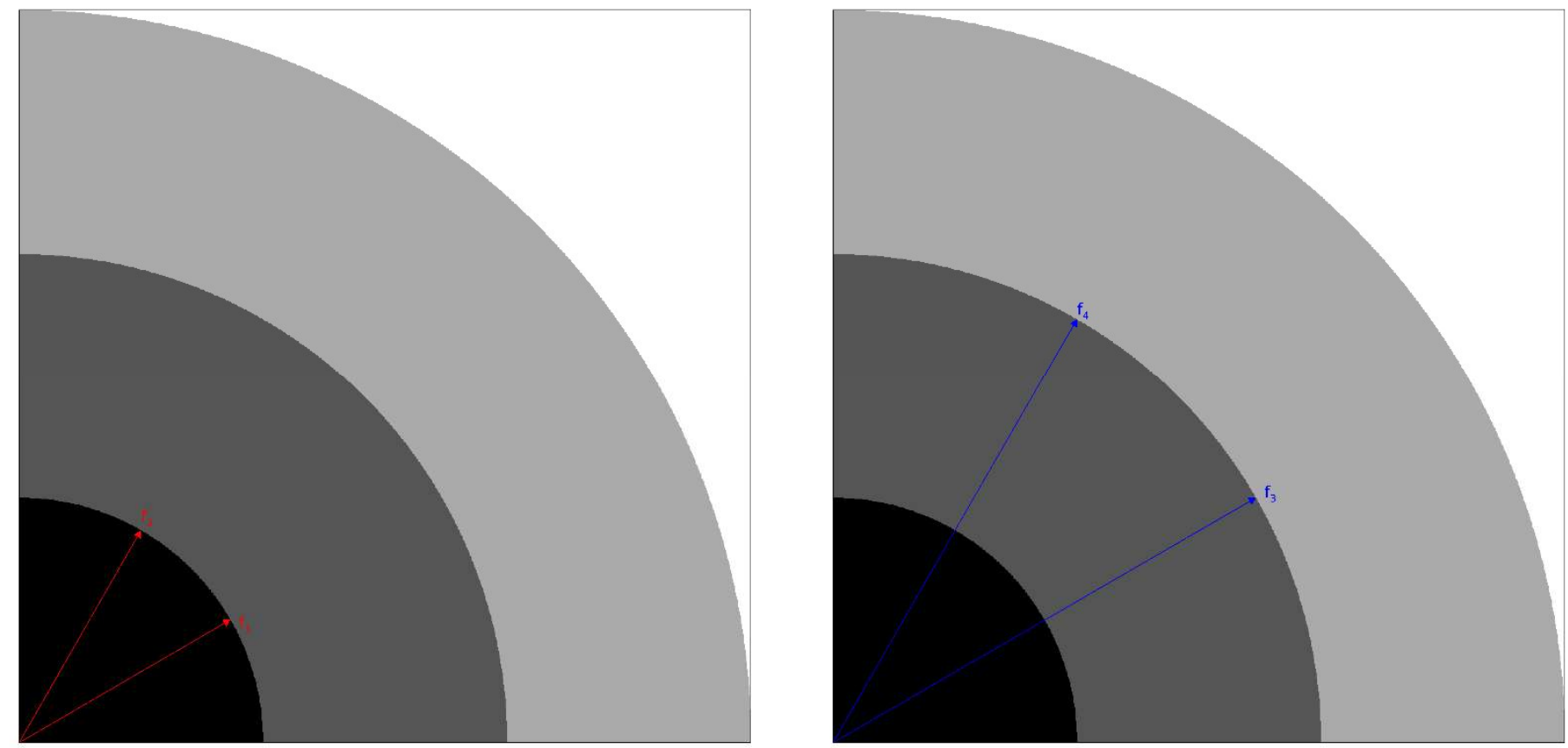

Figure 7: Exploited Views of the $\mathrm{P}_{1}$ Band. These show exploited views of the first quadrant for $\mathrm{P}_{1}$ in the Fourier domain with adjacent bands shown for context. Lines are extended from the origin to each boundary: red for inner boundary and blue for outer boundary. The $\mathrm{f}_{1}$ and $\mathrm{f}_{2}$ points on the inner $\mathrm{P}_{1}$ boundary are shown on the left with $\Delta$ $\approx 0.083$ cycles $/ \mathrm{mm}$ from the origin. The lines connecting these points to the origin are inclined at $\pi / 6$ and $\pi / 3$, respectively. The $f_{3}$ and $f_{4}$ points on the outer boundary are shown in the right pane. These are extensions of $f_{1}$ and $\mathrm{f}_{2}$ along the same directions and are $2 \times \Delta \approx 0.166$ cycles $/ \mathrm{mm}$ from the origin. 

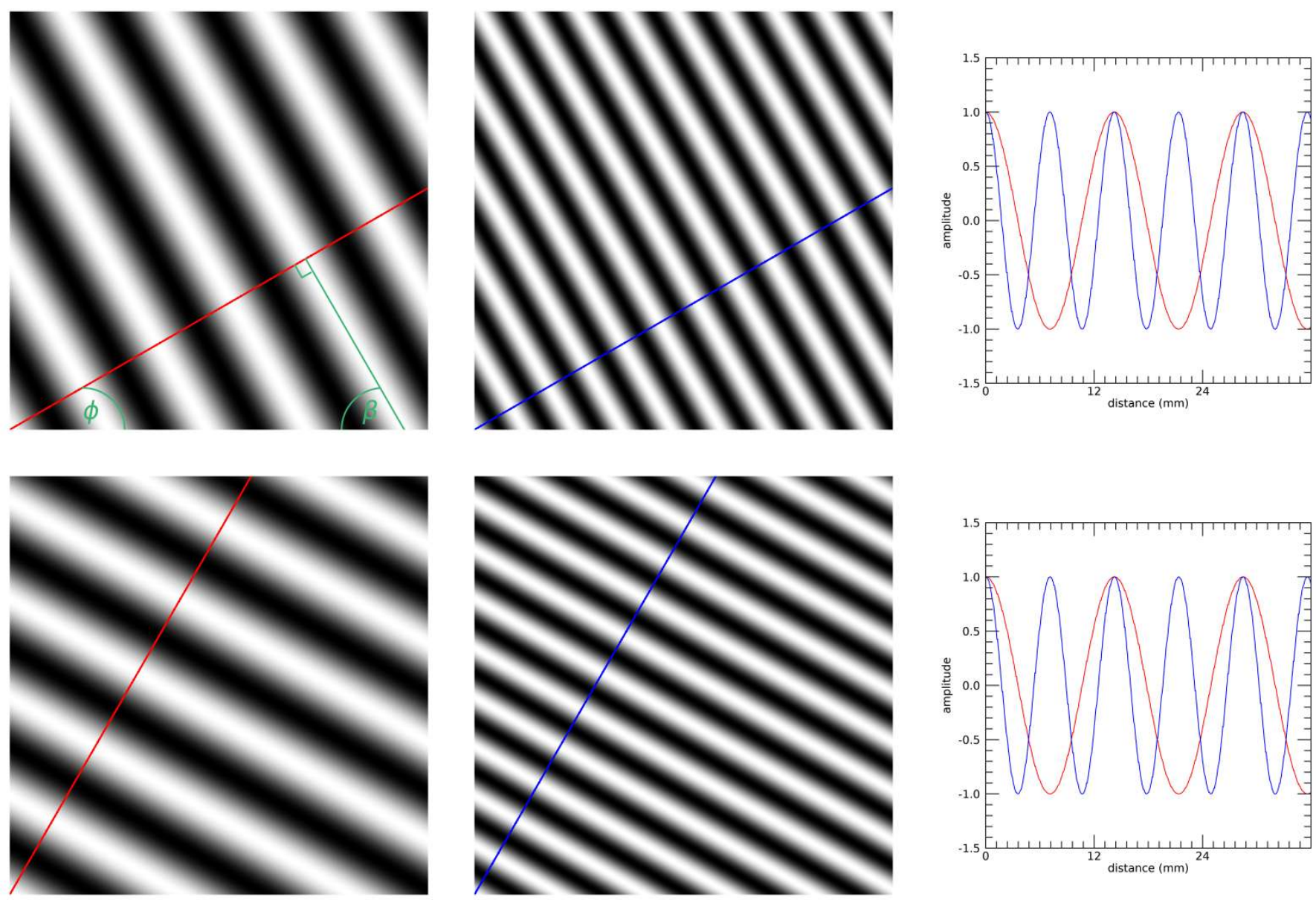

Figure 8: Cosine Corrugations in the Image Domain. These illustrations correspond with the Fourier domain coordinates shown in Figure 7. The left column shows corrugations formed by the $f_{1}$ (top-left) and $f_{2}(-$ bottom) in the image domain (spatial periods $\approx 12 \mathrm{~mm}$ ). The illustrations in the middle column show corrugations formed by $\mathrm{f}_{3}$ (top-middle) and $\mathrm{f}_{4}$ (bottom-middle) in the image domain (spatial periods $\approx 6 \mathrm{~mm}$ ). We use red to indicate lines and profiles related to $f_{1}$ and $f_{2}$ and blue for $f_{3}$ and $f_{4}$. The lines within the corrugations on the top left and middle panes are inclined by $\pi / 6$ above the $\mathrm{x}$-direction and the lines in the bottom left and middle panes by $\pi / 3$. The profiles (cosines) on the right show slices along lines with the same color coding. The top-right plots show these profiles along the respective lines for $f_{1}$ and $f_{2}$ corrugations. The bottom-left plots show profiles along the respective lines for $\mathrm{f}_{3}$ and $\mathrm{f}_{4}$. The top-left pane (green) shows the direction of the corrugation, $\phi$, and $\beta$. 

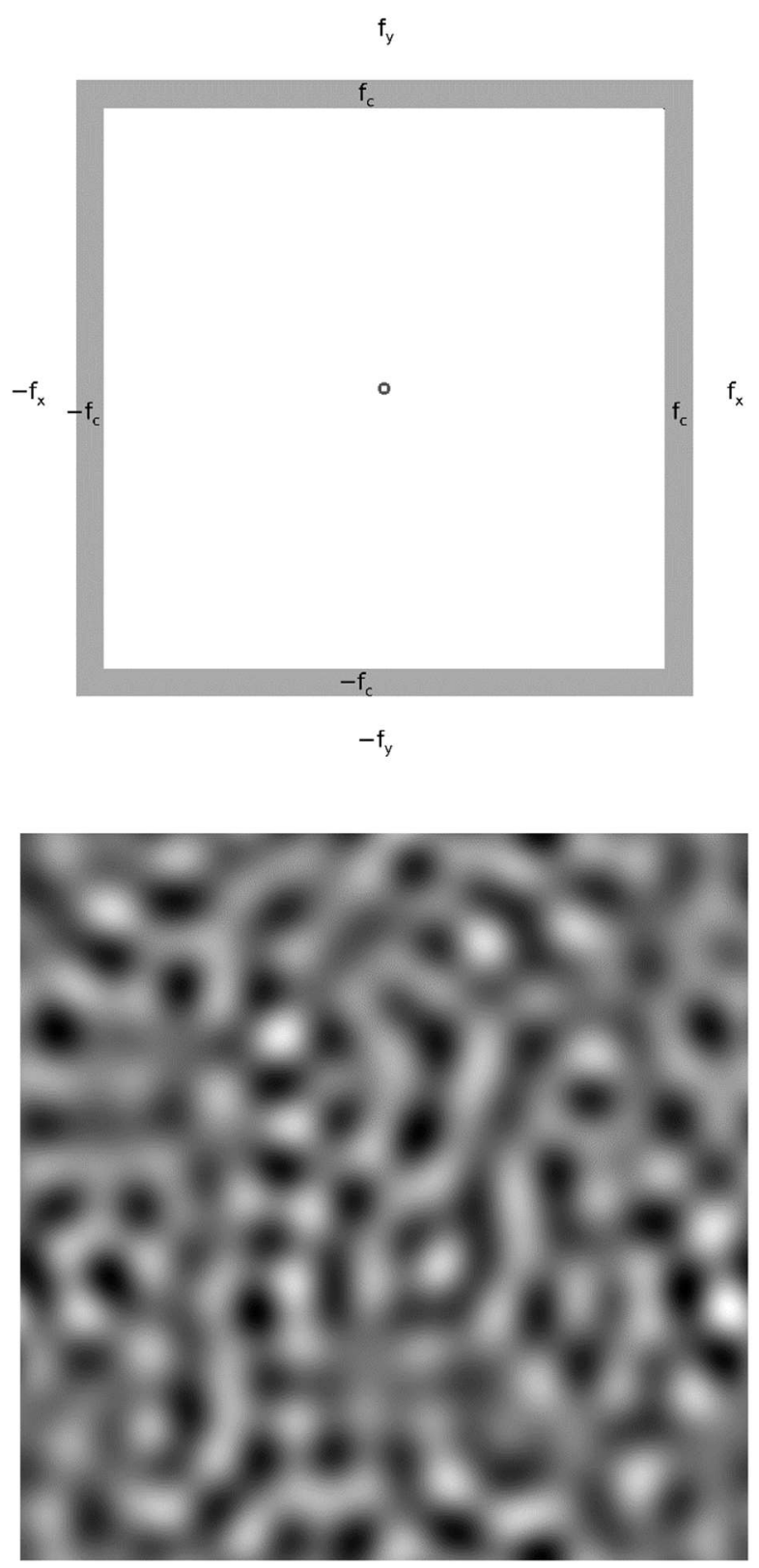

Figure 9: The $\mathrm{m}=1$ Radial Band in Both Domains. The Fourier radial band for $\mathrm{m}=1$ with $\mathrm{n}=86$ is shown in the top pane. The coordinate axes have been suppressed for viewing purposes. The texture from the respective filtering process is shown in the bottom pane. 

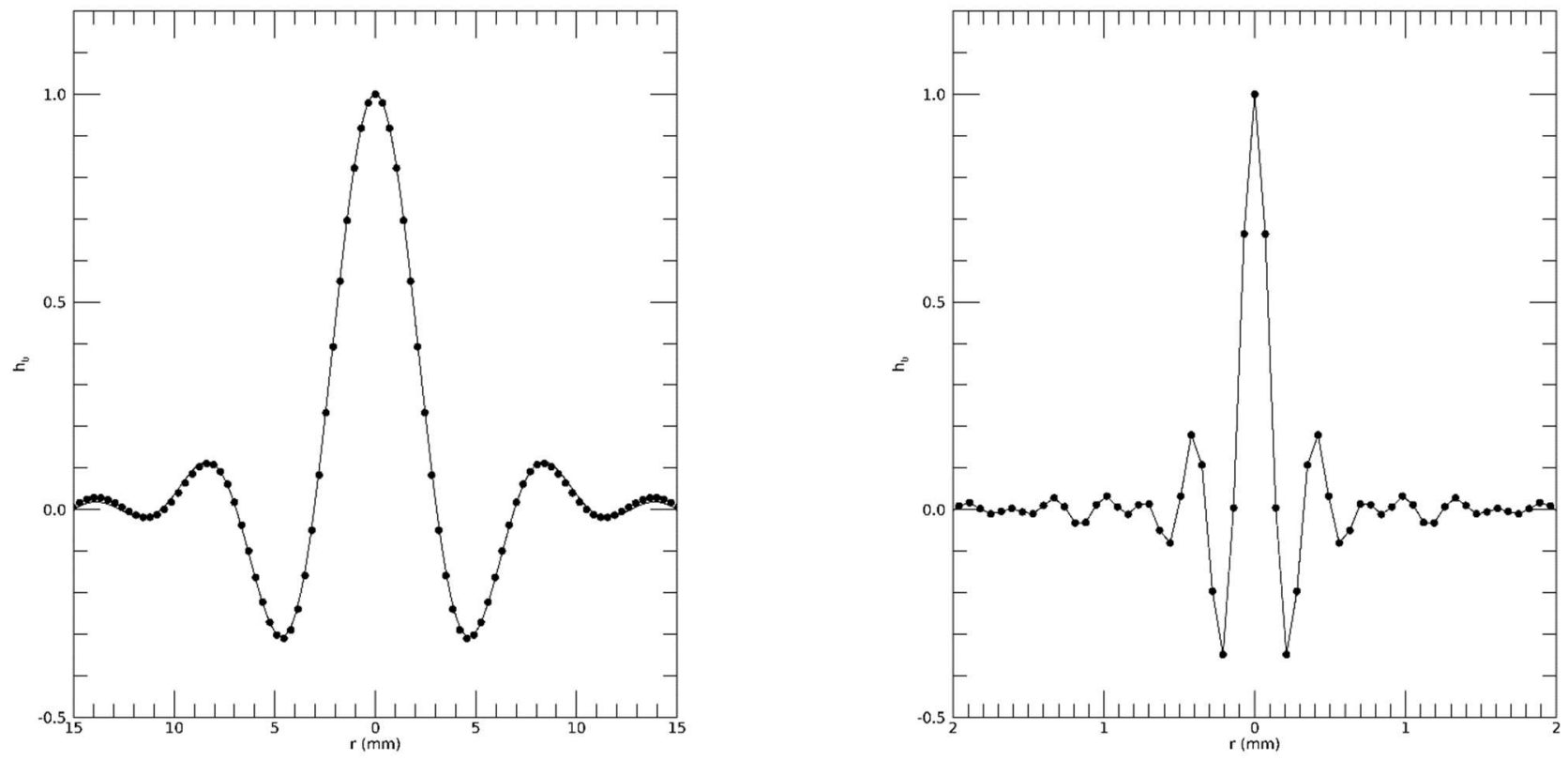

Figure 10: Radial Bandpass Filter Kernel Profiles. These plots illustrate the spatial filter kernels for a narrow low-frequency band $[\Delta, 2 \times \Delta]$ (left) corresponding with Figure 8 and a wider more central band $[25 \times \Delta, 39 \times \Delta]$ (right). The numerically derived kernels (dots) are compared with the theoretical plots (solid) derived from Eq. [9]. These are two-dimensional radially symmetric non-separable kernels. Kernels were normalized to unity at the origin. 

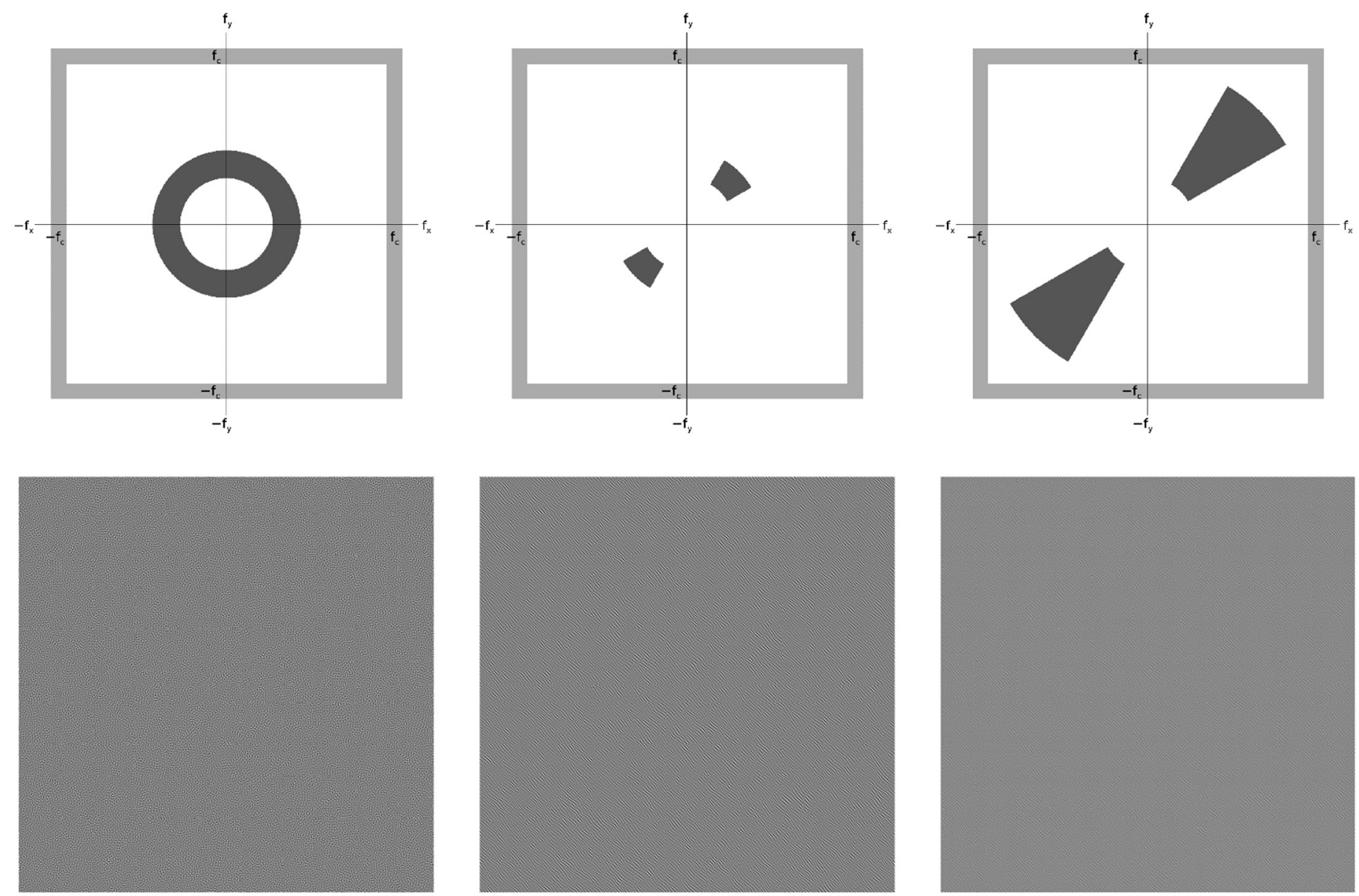

Figure 11: Radial Bandpass Filters and Texture with Directional Attributes. The top left-pane shows a more general bandpass filter in the Fourier domain and the bottom left-pane shows texture it generates; the texture does not have directional attributes. The top middle-pane shows a filter with the same radial bandwidth restricted to an arc; the corresponding texture in the bottom middle-pane now exhibits directional attributes. The top right-pane shows a wider radial bandpass filter with lower radial cutoff equivalent to the upper cutoff the filter shown in the upper middle pane restricted to the same arc; the striations exhibited the same directional attributes as the middlepane filter output with finer detail because of the radial bandwidth increase. 


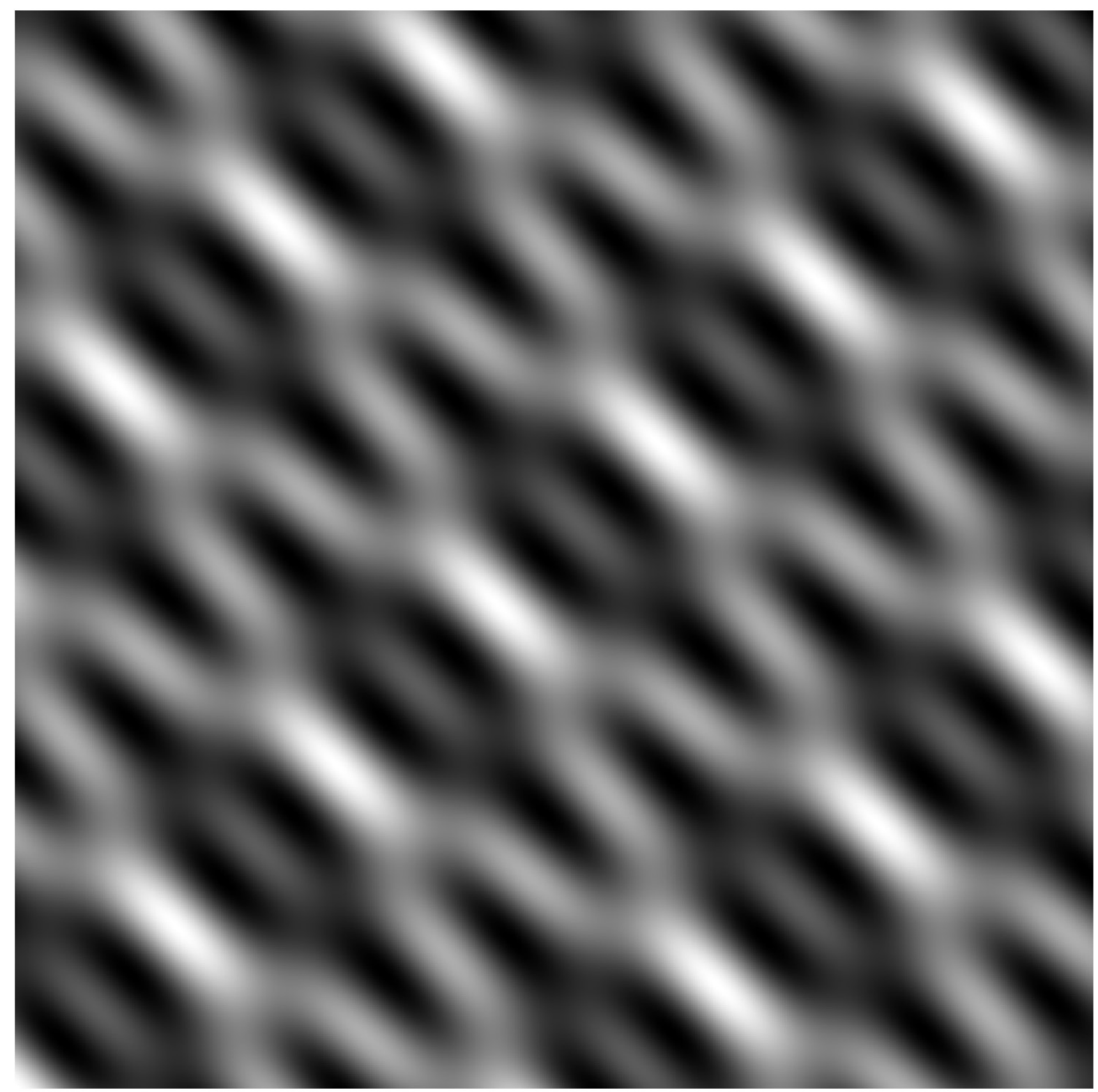

Figure 11: Composite Image. This shows the structure created by summing four cosine corrugation images with equal weights. Striations show a definite orientation, roughly in the northwest (diagonal) direction, because the constituent corrugations are restricted to two directions. 

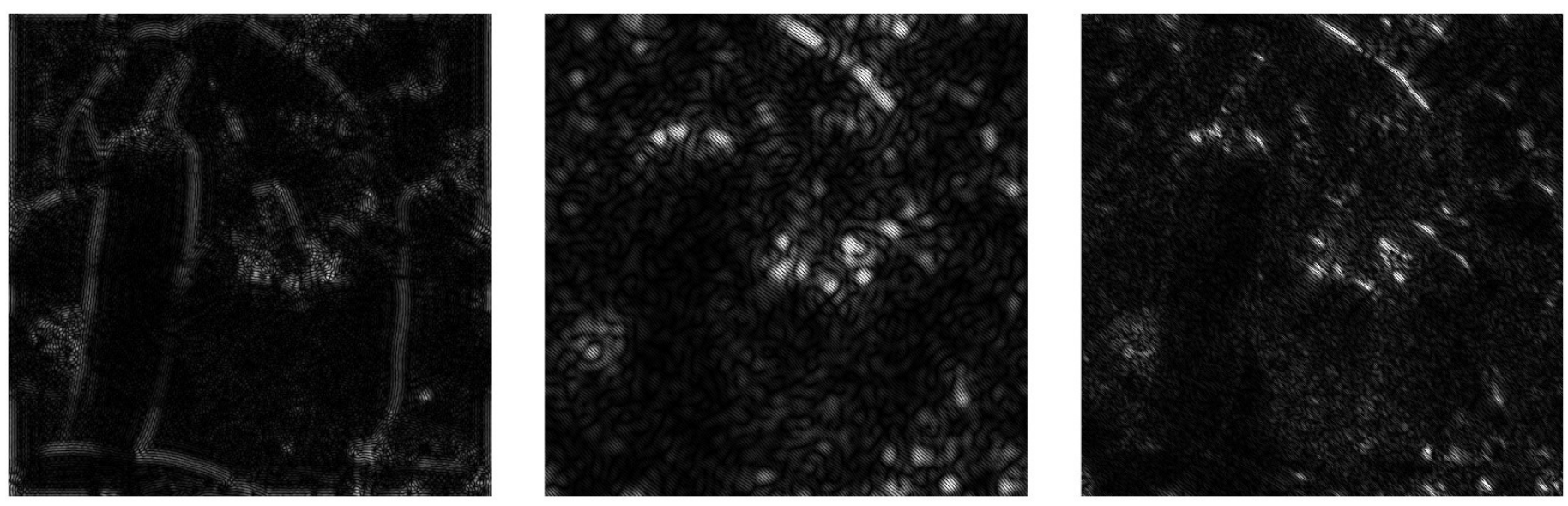

Figure 13: Bandpass Filtering Examples Using the Peppers Image. The pepper image was used as the input to the filters three filters shown in Figure 12. The filter outputs are shown in the same order as in Figure 12; these are absolute values images displayed with over contrasting for viewing purposes. 


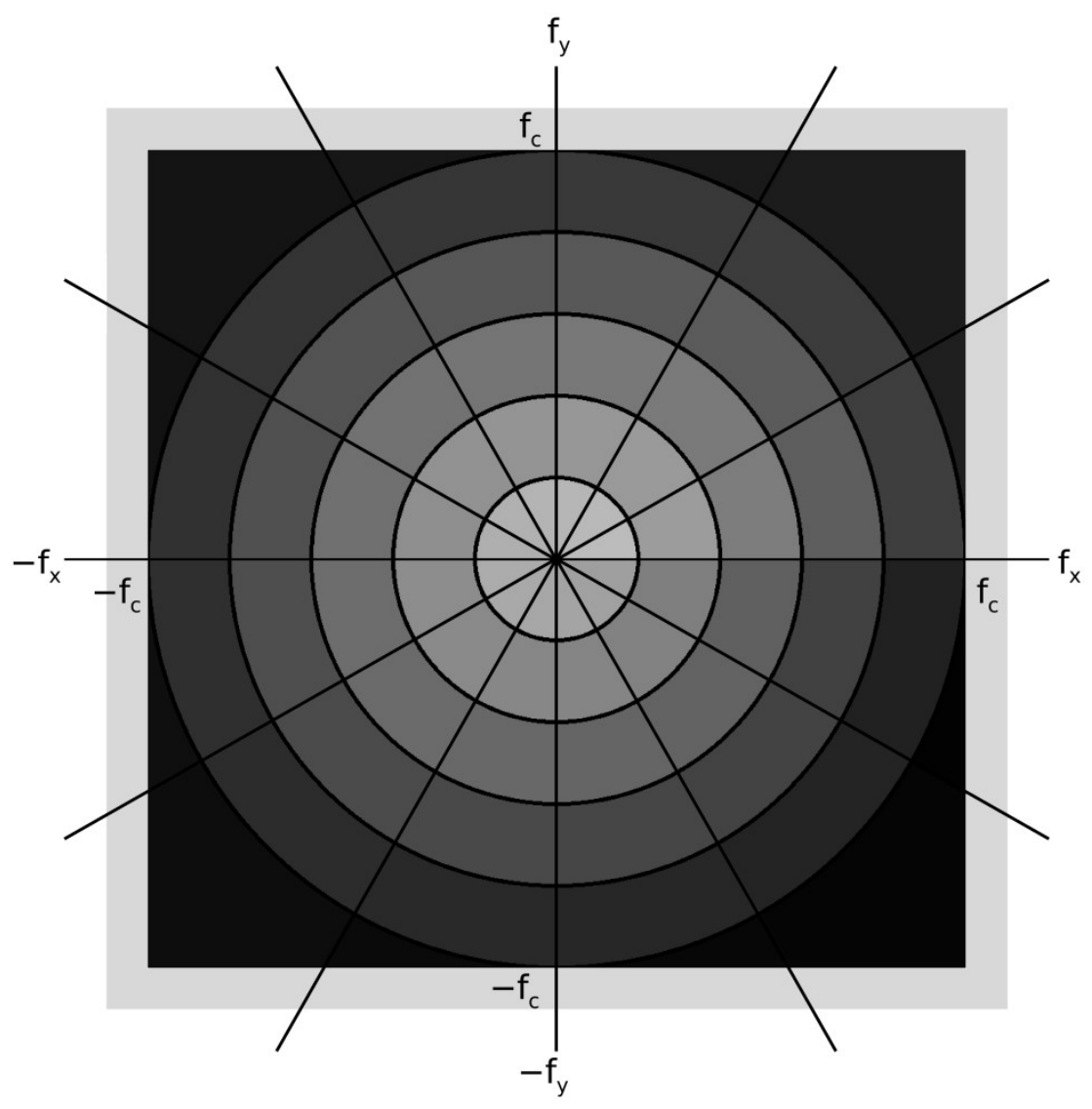

Figure 14: Radial and Arc Bandwidths. This the Fourier plane is divided into both radial and arc bandwidths. In this coarse example, we used $n=5$ radial divisions and $k=6$ angular divisions. Angles are measured in a counterclockwise direction from the positive $\mathrm{f}_{\mathrm{x}}$ axis. 


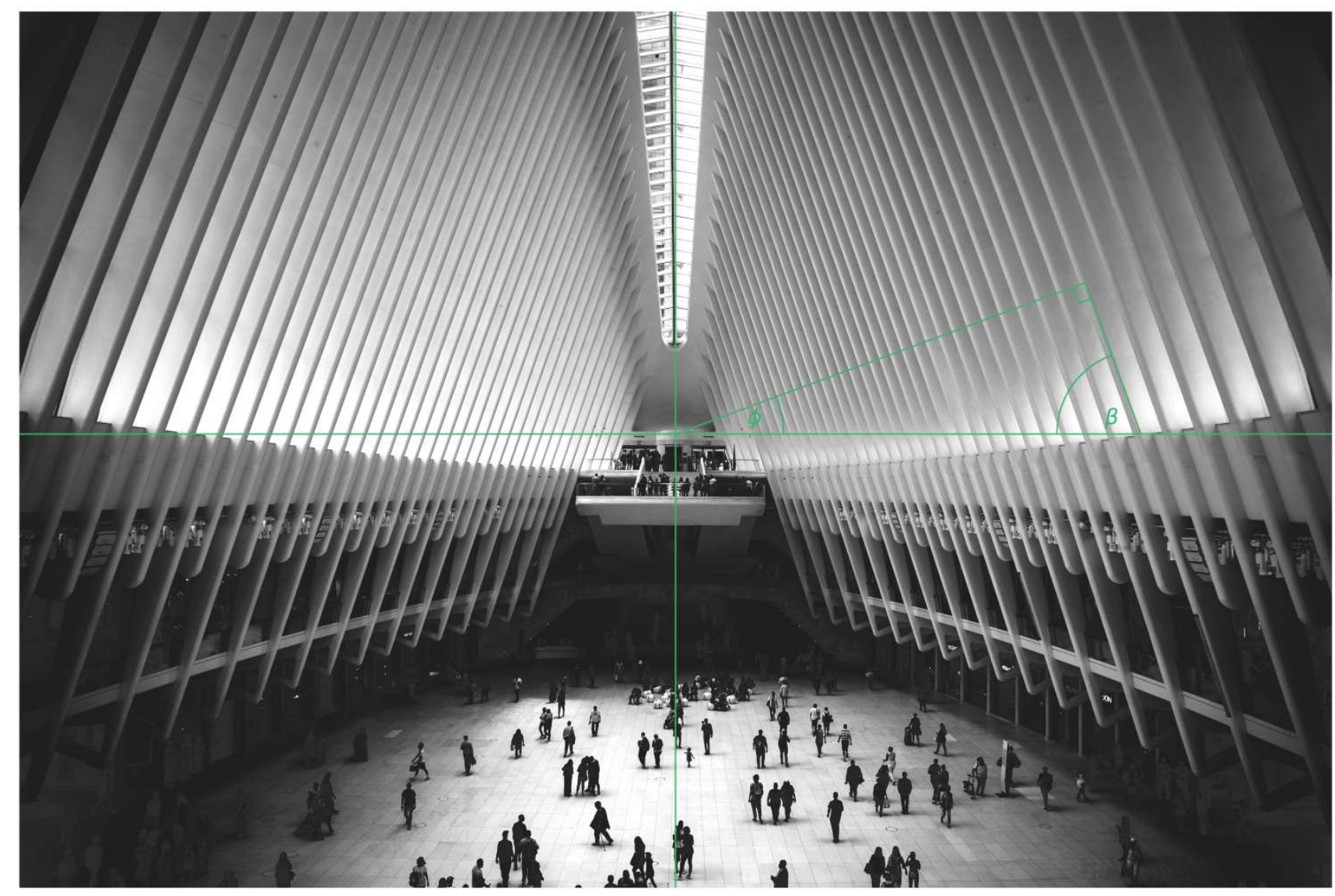

Figure 15: Crowd Walking on Alley. This image has distinct linear structures. Cartesian coordinate axes and a right triangle (green) were superimposed on this image to aid the illustration. The radial line segment from the origin (center of the image) intersects the pronounced linear structure at $\frac{\pi}{2}$ (90 degrees): $\phi \approx 0.11 \times \pi$ (approximately 20 degrees) and $\beta \approx 0.39 \pi$ (approximately 70 degrees). 

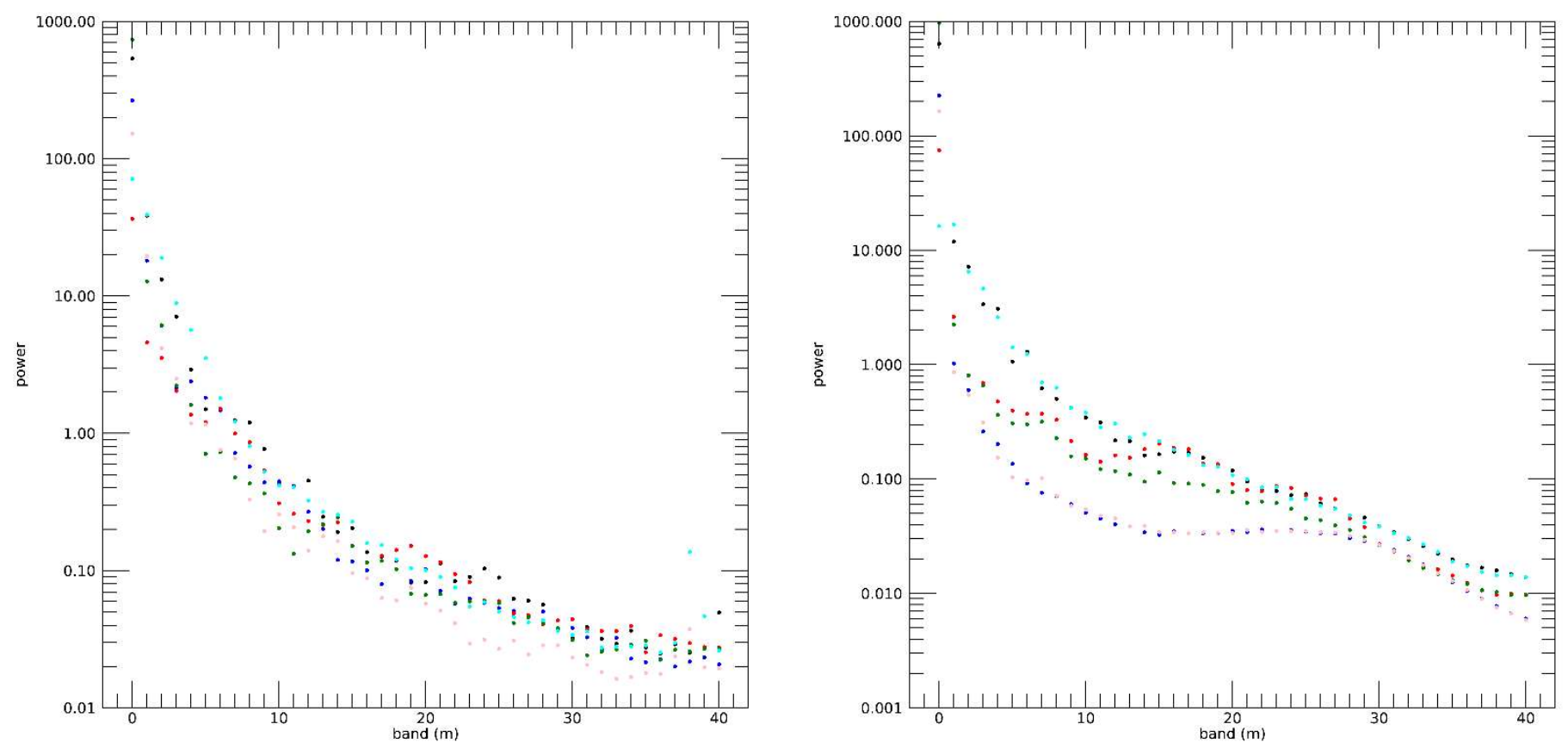

Figure 16: Angular Dependent $P_{m l}$ Plots for the Peppers (left) and Crowd Walk on Alley (right) images. This shows the $\mathrm{P}_{\mathrm{m} 1}$ (black), $\mathrm{P}_{\mathrm{m} 2}$ (blue), $\mathrm{P}_{\mathrm{m} 3}$ (red), $\mathrm{P}_{\mathrm{m} 4}$ (green), $\mathrm{P}_{\mathrm{m} 5}$ (pink), and, $\mathrm{P}_{\mathrm{m} 6}$ (cyan) plots on a logarithmic scale corresponding with these arcs: $\left[0, \frac{\pi}{6}\right),\left[\frac{\pi}{6}, \frac{\pi}{3}\right),\left[\frac{\pi}{3}, \frac{\pi}{2}\right),\left[\frac{\pi}{2}, \frac{2 \pi}{3}\right)\left[\frac{2 \pi}{3}, \frac{5 \pi}{6}\right),\left[\frac{5 \pi}{6}, \pi\right)$, respectively. 

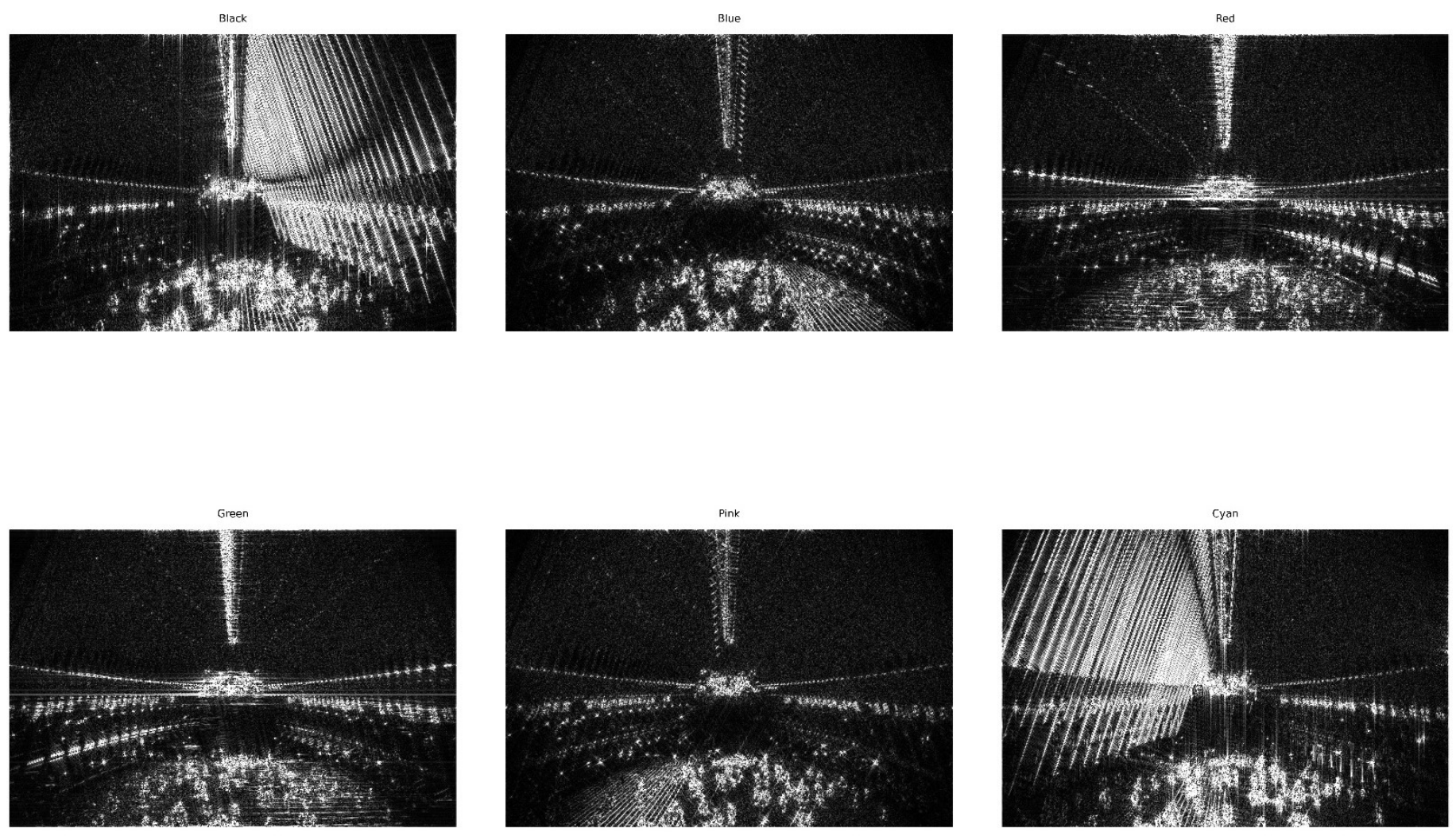

Figure 17: Filtered CWA images for filter (1): $m=5-10$ radial bands and 6 angular bands. The images are ordered according to the angular bands defined in Figure 16. The linear structure defined in Figure 15 is observable in the black (top-left) and cyan bands (bottom-right) and blocked for the other angular bands. Also note, the center ridge is visible in the red (top-right) and green (bottom-left) bands. 

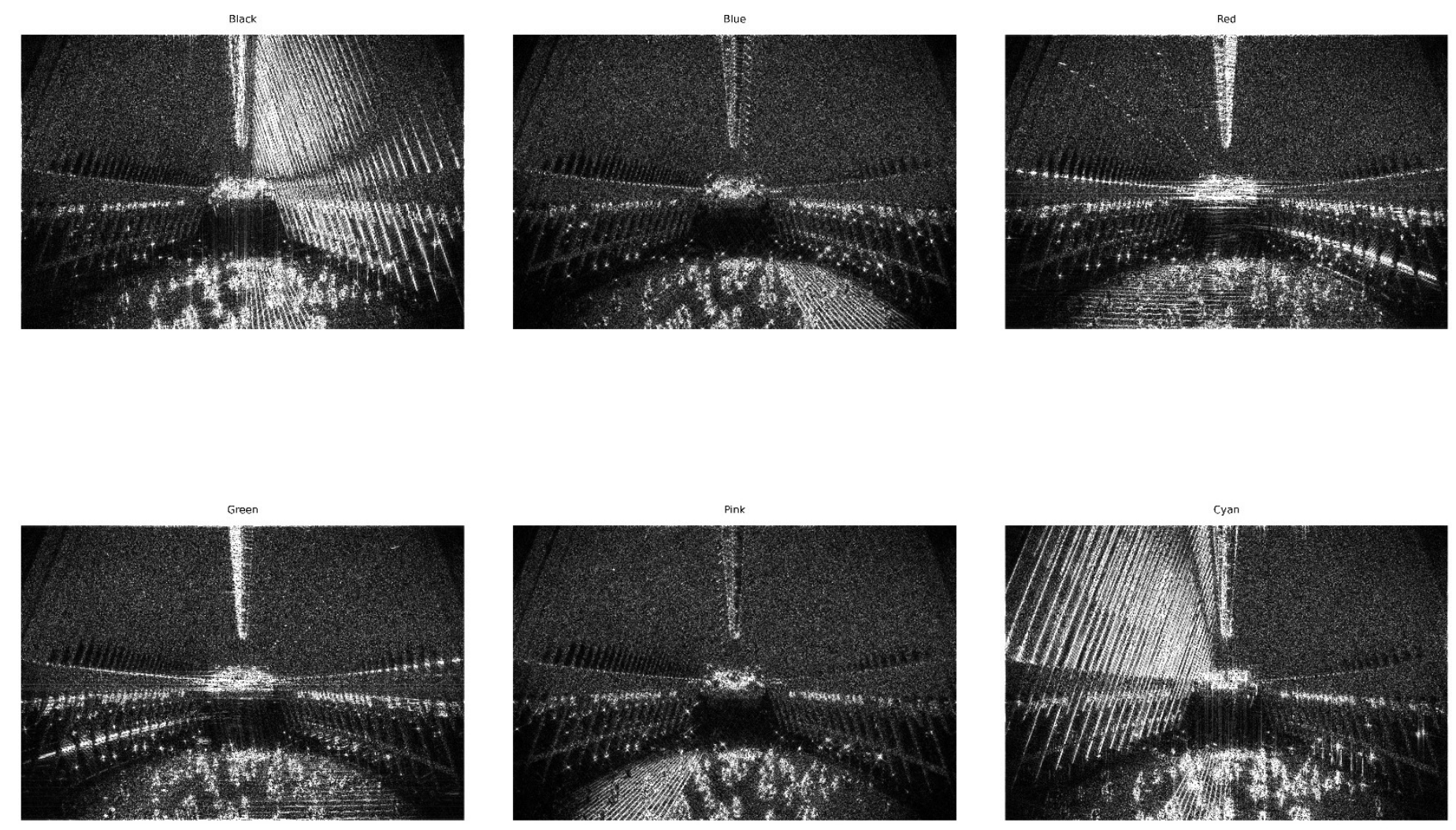

Figure 18: Filtered CWA images for $\mathrm{m}=10-15^{\text {th }}$ radial bands and 6 angular bands. The images are ordered according to the angular bands defined in Figure 16. The linear structure defined in Figure 15 is observable in the black (top-left) and cyan bands (bottom-right) and blocked for the other angular bands. Also note, the center ridge is visible in the red (top-right) and green (bottom-left) bands. 

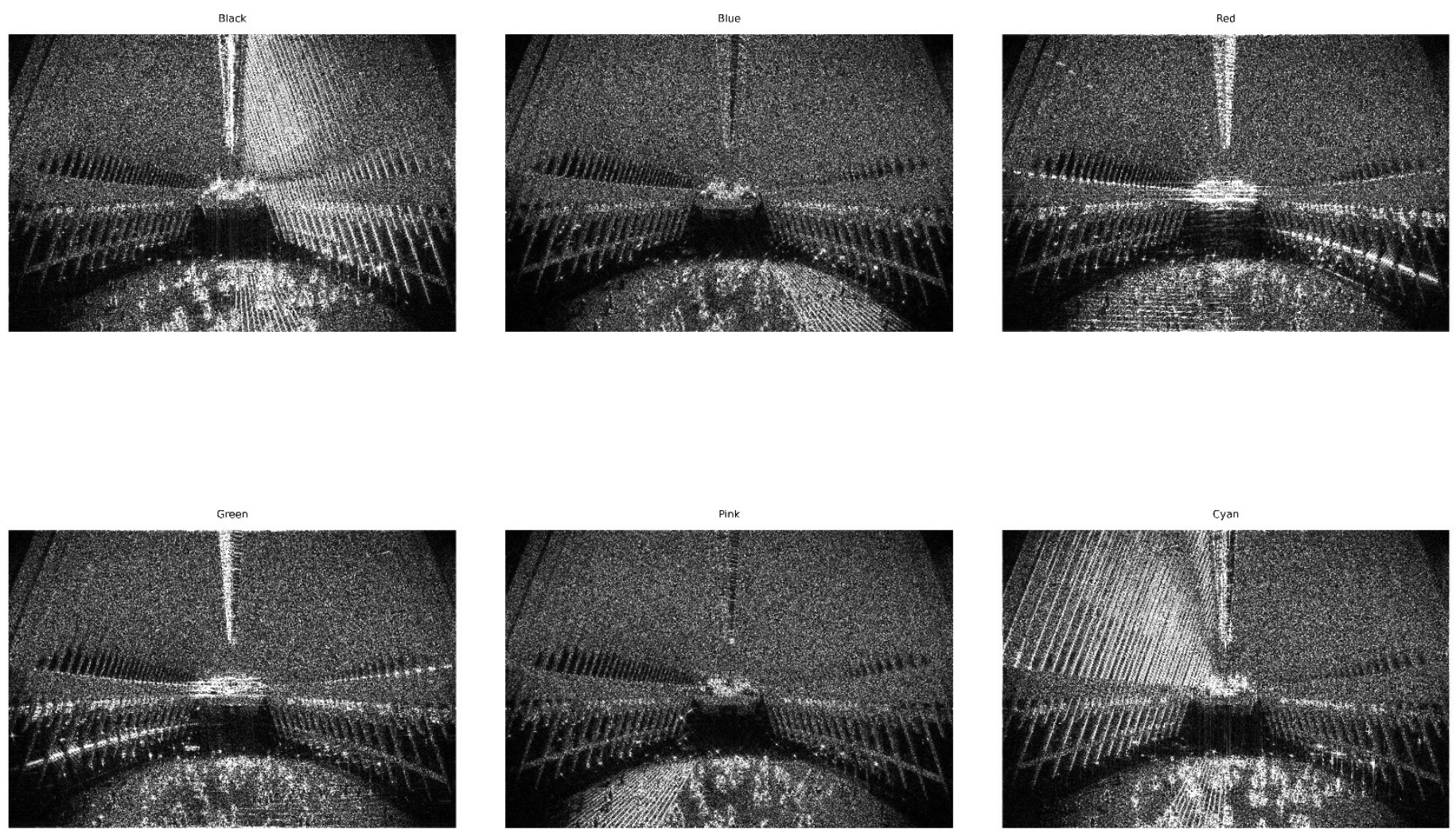

Figure 19: Filtered CWA images for the $\mathrm{m}=15-20^{\text {th }}$ radial bands and 6 angular bands. The images are ordered according to the angular bands defined in Figure 16. The linear structure defined in Figure 15 is observable in the black (top-left) and cyan bands (bottom-right) and blocked for the other angular bands. Also note, the center ridge is visible, although fading, in the red (top-right) and green (bottom-left). 

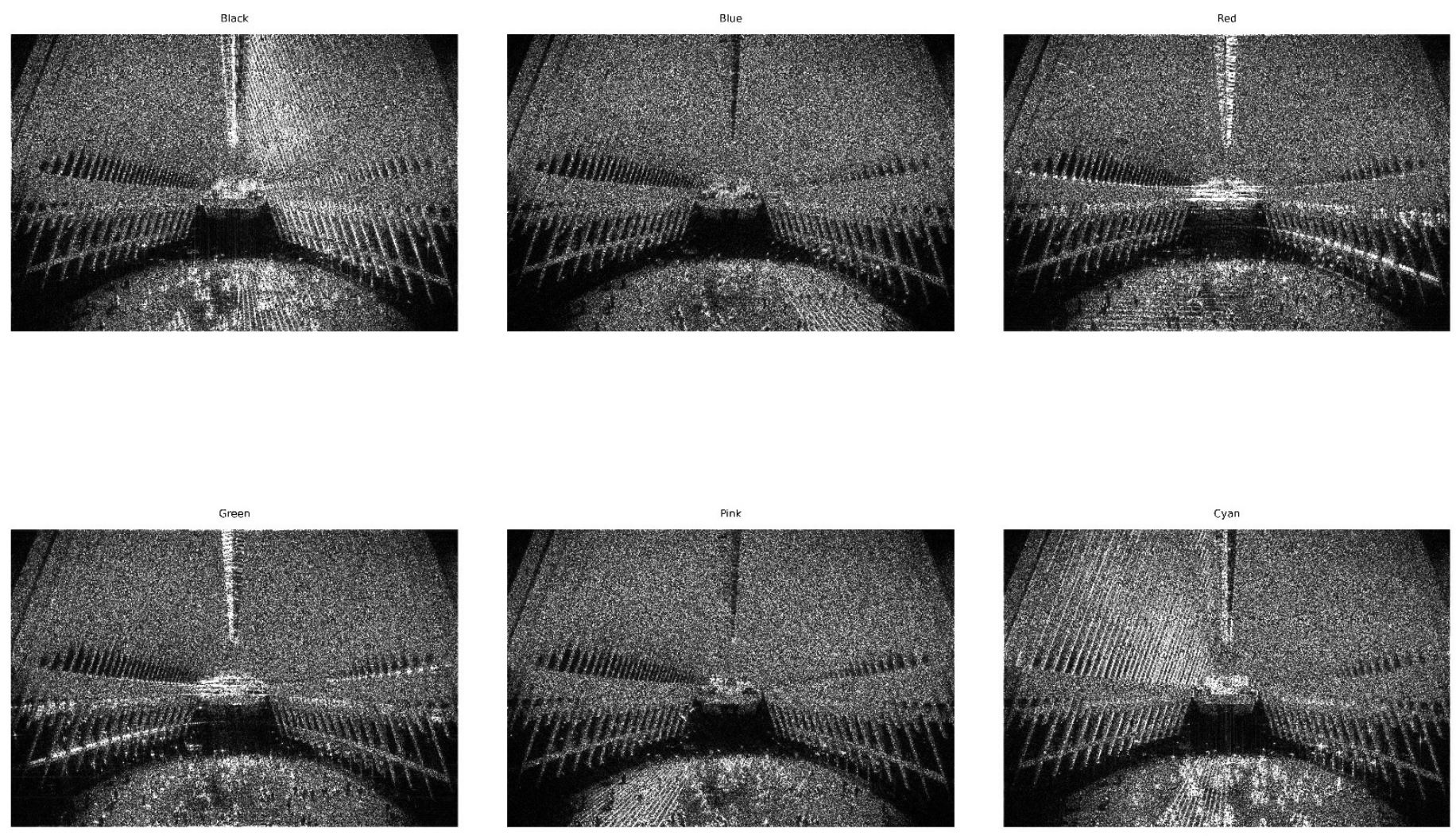

Figure 20: Filtered CWA images for $m=20-25^{\text {th }}$ radial bands and 6 angular bands. The images are ordered according to the angular bands defined in Figure 16. The linear structure defined in Figure 15 is observable in the black (top-left) and cyan bands (bottom-right) and blocked for the other angular bands. Also note, the center ridge is visible in the red (top-right) and green (bottom-left) bands. 

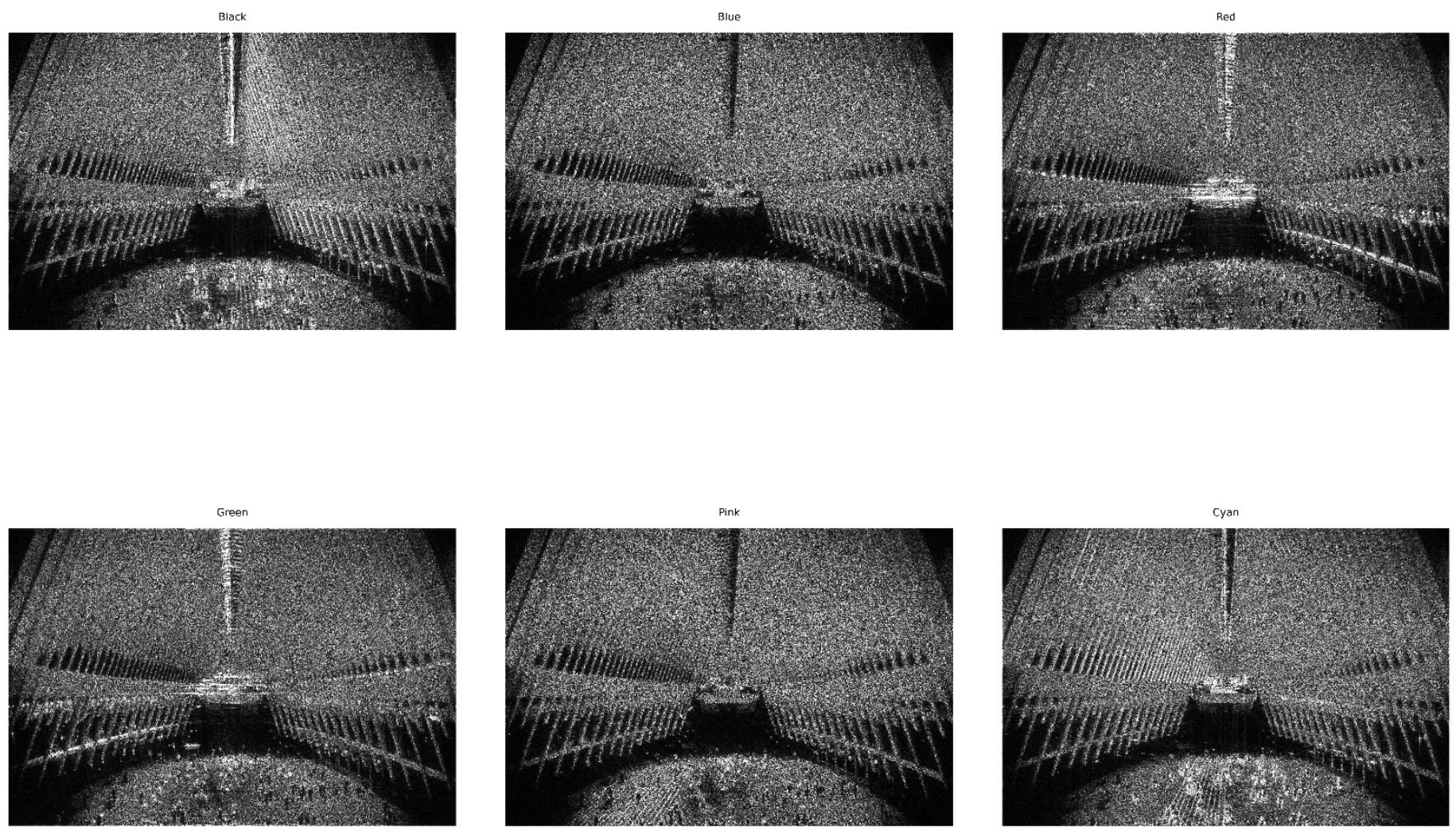

Figure 21: Filtered CWA images for $25-30^{\text {th }}$ radial bands and 6 angular bands. The images are ordered according to the angular bands defined in Figure 16. The linear structure defined in Figure 15 is observable in the black (top-left) and cyan bands (bottom-right) and blocked for the other angular bands. Also note, the center ridge is visible in the red (top-right) and green (bottom-left) bands. 

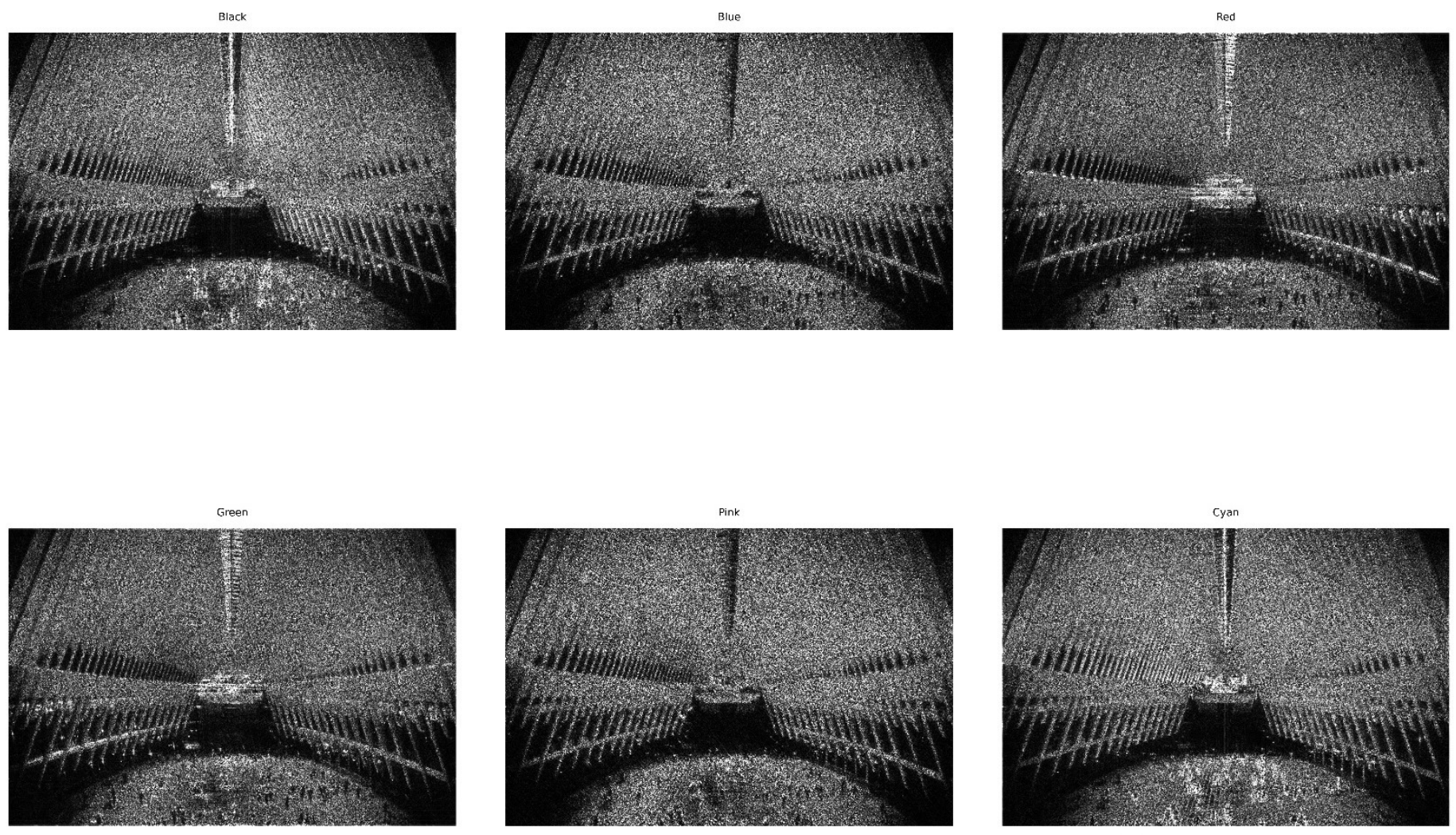

Figure 22: Filtered CWA images for $\mathrm{m}=30-35^{\text {th }}$ radial bands and 6 angular bands. The images are ordered according to the angular bands defined in Figure 16. The linear structure defined in Figure 15 is observable in the black (top-left) and cyan bands (bottom-right) and blocked for the other angular bands. Also note, the center ridge is visible in the red (top-right) and green (bottom-left) bands. 

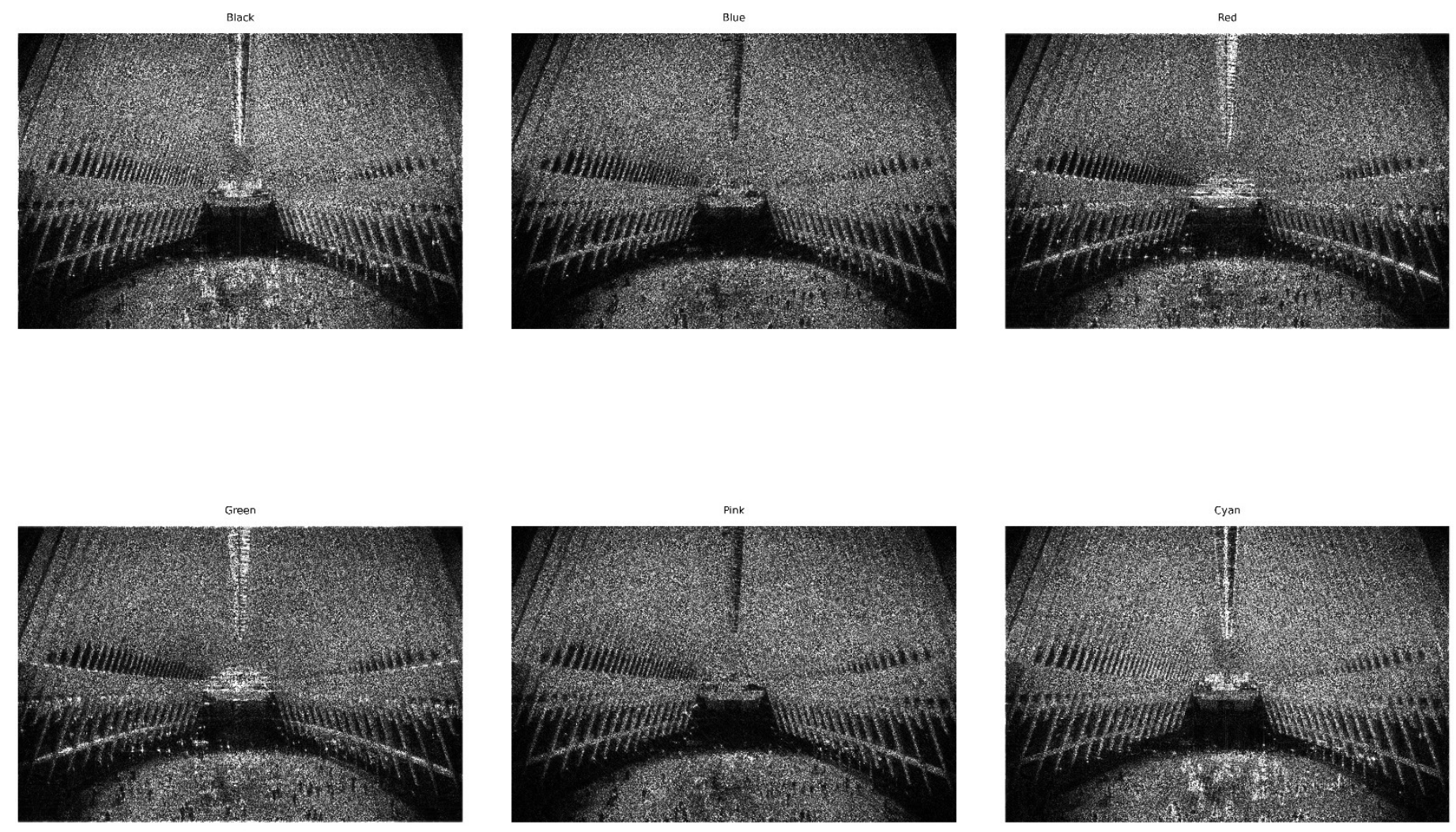

Figure 23: Filtered CWA images for $\mathrm{m}=35-40^{\text {th }}$ radial bands and 6 angular bands. The images are ordered according to the angular bands defined in Figure 16. The linear structure defined in Figure 15 is observable in the black (top-left) and cyan bands (bottom-right) and blocked for the other angular bands. Also note, the center ridge is visible in the red (top-right) and green (bottom-left) bands. 

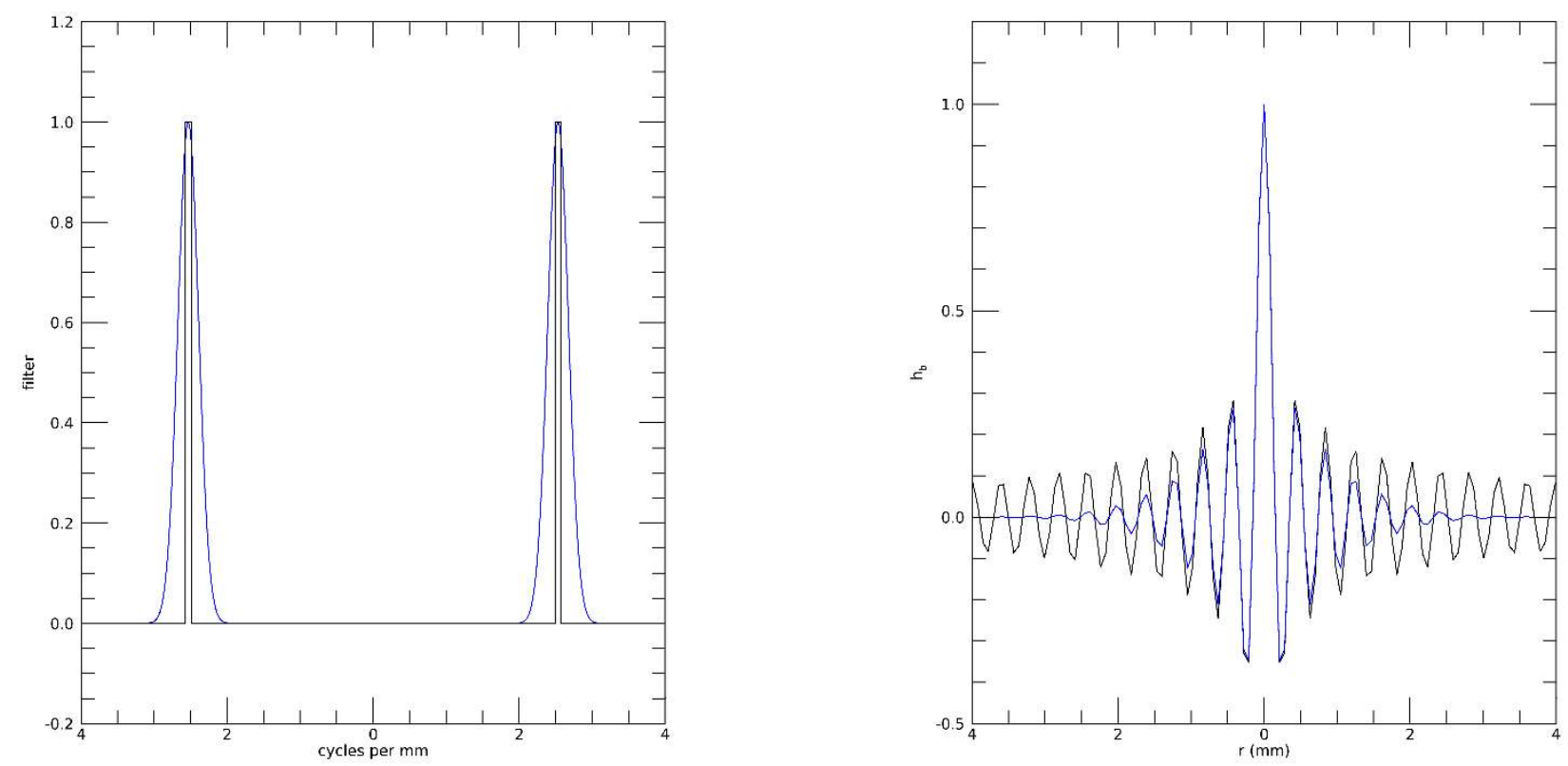

Figure 24: Radial Bandpass Filter Modification. The pane on the left shows the profile for the $\mathrm{m}=30$ band filter in the Fourier domain (black) compared with the modified filter's profile (blue). The corresponding profiles for the spatial domain kernels are compared in the right pane: band filter (black) and modification (blue). 


\section{References}

[1] R. M. Rangayyan, Biomedical Image Analysis. Boca Raton, FL: CRC press, 2004.

[2] S. Renukalatha and K. Suresh, "A review on biomedical image analysis," Biomedical Engineering: Applications, Basis and Communications, vol. 30, no. 04, p. 1830001, 2018.

[3] J. S. Suri, D. Wilson, and S. Laxminarayan, Handbook of biomedical image analysis. Springer Science \& Business Media, 2005.

[4] I. Dronova, "Object-based image analysis in wetland research: A review," Remote Sensing, vol. 7, no. 5, pp. 63806413, 2015.

[5] K. E. Joyce, S. E. Belliss, S. V. Samsonov, S. J. McNeill, and P. J. Glassey, "A review of the status of satellite remote sensing and image processing techniques for mapping natural hazards and disasters," Progress in physical geography, vol. 33, no. 2, pp. 183-207, 2009.

[6] C. Duchesne, J. Liu, and J. MacGregor, "Multivariate image analysis in the process industries: A review," Chemometrics and Intelligent Laboratory Systems, vol. 117, pp. 116-128, 2012.

[7] J. I. Olszewska, "Automated face recognition: challenges and solutions," Pattern Recognition-Analysis and Applications, pp. 59-79, 2016.

[8] H. Cai et al., "Breast Microcalcification Diagnosis Using Deep Convolutional Neural Network from Digital Mammograms," (in English), Computational and mathematical methods in medicine, vol. 2019, p. 2717454, 2019.

[9] A. A. Mohamed, W. A. Berg, H. Peng, Y. Luo, R. C. Jankowitz, and S. Wu, "A deep learning method for classifying mammographic breast density categories," (in English), Medical physics, vol. 45, no. 1, pp. 314-321, 2018.

[10] A. Gastounioti, E. F. Conant, and D. Kontos, "Beyond breast density: a review on the advancing role of parenchymal texture analysis in breast cancer risk assessment," Breast Cancer Research, vol. 18, no. 1, p. 91, 2016.

[11] X. Xie, "A review of recent advances in surface defect detection using texture analysis techniques," ELCVIA: electronic letters on computer vision and image analysis, vol. 7, no. 3, pp. 1-22, 2008.

[12] S. Chambon and J.-M. Moliard, "Automatic road pavement assessment with image processing: review and comparison," International Journal of Geophysics, vol. 2011, 2011.

[13] S. Kabir, "Image Processing in Concrete Applications: Review and Prospective," in 2nd International Structural Specialty Conference on Partnership for Innovation: Instrumentation and Monitoring of Structures, CSCE Annual Conference, Quebec City, 2008.

[14] v. A. Van der Schaaf and J. v. van Hateren, "Modelling the power spectra of natural images: statistics and information," Vision research, vol. 36, no. 17, pp. 2759-2770, 1996.

[15] D. L. Ruderman, "Origins of scaling in natural images," Vision research, vol. 37, no. 23, pp. 3385-3398, 1997.

[16] V. A. Billock, G. C. de Guzman, and J. S. Kelso, "Fractal time and $1 / \mathrm{f}$ spectra in dynamic images and human vision," Physica D: Nonlinear Phenomena, vol. 148, no. 1-2, pp. 136-146, 2001.

[17] A. Manduca et al., "Texture features from mammographic images and risk of breast cancer," Cancer Epidemiol Biomarkers Prev, vol. 18, no. 3, pp. 837-45, Mar 2009.

[18] J. Kalpathy-Cramer et al., "Radiomics of Lung Nodules: A Multi-Institutional Study of Robustness and Agreement of Quantitative Imaging Features," (in English), Tomography (Ann Arbor, Mich ), vol. 2, no. 4, pp. 430-437, 2016.

[19] A. Kassner and R. E. Thornhill, "Texture analysis: a review of neurologic MR imaging applications," (in English), AJNR American journal of neuroradiology, vol. 31, no. 5, pp. 809-16, 2010.

[20] J. J. Heine, S. R. Deans, R. P. Velthuizen, and L. P. Clarke, "On the statistical nature of mammograms," (in eng), Medical physics, vol. 26, no. 11, pp. 2254-65, Nov 1999.

[21] J. J. Heine and R. P. Velthuizen, "A statistical methodology for mammographic density detection," Med Phys, vol. 27, no. 12, pp. 2644-51, Dec 2000.

[22] J. J. Heine and R. P. Velthuizen, "Spectral analysis of full field digital mammography data," Med Phys, vol. 29 , no. 5, pp. 647-61, May 2002. 
[23] J. Huang and D. Turcotte, "Fractal mapping of digitized images: application to the topography of Arizona and comparisons with synthetic images," Journal of Geophysical Research: Solid Earth, vol. 94, no. B6, pp. 7491-7495, 1989.

[24] C. Redies, J. Hasenstein, and J. Denzler, "Fractal-like image statistics in visual art: similarity to natural scenes," Spatial vision, vol. 21, no. 1-2, pp. 137-148, 2007.

[25] M. Schroeder, Fractals, chaos, power laws: Minutes from an infinite paradise. New York: W.H. Freeman and Company, 1991.

[26] E. E. E. Fowler, C. Hathaway, F. Tillman, R. Weinfurtner, T. A. Sellers, and J. Heine, "Spatial correlation and breast cancer risk," Biomedical Physics \& Engineering Express, vol. 5, no. 4, p. 045007, 2019/05/22 2019.

[27] E. E. E. Fowler, A. Smallwood, C. Miltich, J. Drukteinis, T. A. Sellers, and J. Heine, "Generalized breast density metrics," (in English), Physics in Medicine and Biology, vol. 64, no. 1, Jan 2019.

[28] E. O. Brigham, The fast Fourier transform and its applications (Prentice-Hall signal processing series). Englewood Cliffs, N.J.: Prentice Hall, 1988, pp. xvi, 448 p.

[29] R. N. Bracewell, Two-dimensional imaging (Prentice-Hall signal processing series). Englewood Cliffs, N.J.:

Prentice Hall, 1995, pp. xiv, 689 p.

[30] D. Slepian, "On bandwidth," Proceedings of the IEEE, vol. 64, no. 3, pp. 292-300, 1976.

[31] Photograph by Adam Bentley titled Crowd Walking on Alley, archived at https://unsplash.com/photos/kbUfl3Apb6E.

[32] G. R. Osche, Optical detection theory for laser applications (Wiley series in pure and applied optics). Hoboken, N.J.: Wiley-Interscience, 2002, pp. xii, 412 p.

[33] R. N. Bracewell, Fourier Analysis and Imaging Springer Science \& Business Media, LCC, 2006.

[34] I. Daubechies, Ten lectures on wavelets (CBMS-NSF regional conference series in applied mathematics, no. 61). Philadelphia, Pa.: Society for Industrial and Applied Mathematics, 1992, pp. xix, 357 p.

[35] J. J. Heine, S. R. Deans, D. K. Cullers, R. Stauduhar, and L. P. Clarke, "Multiresolution probability analysis of grayscaled images," Journal of the Optical Society of America A, vol. 15, no. 5, pp. 1048-1058, 1998.

[36] J. J. Heine, S. R. Deans, D. Gangadharan, and L. P. Clarke, "Multiresolution analysis of two-dimensional 1/f processes: approximation methods for random variable transformations," Optical Engineering, vol. 38, p. 1505, 1999.

[37] R. N. Bracewell, The Fourier transform and its applications, 2nd ed. (McGraw-Hill series in electrical engineering Circuits and systems). New York: McGraw-Hill, 1986, pp. xx, 474 p.

[38] I. S. Gradshteyn and I. M. Ryzhik, A. Jeffrey, Ed. Table of Integrals, Series, and Products, Fifth Edition ed. Boston, MA: Academic Press, Inc., 1994. 